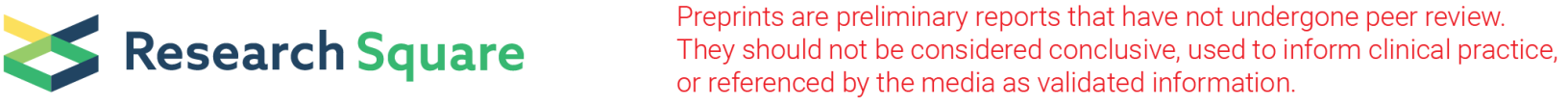 \\ Knowledge Mapping of Migraine: a Bibliometric Analysis (2000- 2019)
}

\section{Yanqing Zhao}

Shanghai Municipal Hospital of Traditional Chinese Medicine

Wen-tao Li ( $D$ lwt1132@163.com )

Encephalopathy Department, Shanghai Municipal Hospital of Traditional Chinese Medicine

Research article

Keywords: Knowledge Mapping, migraine, CiteSpace, bibliometric analysis

Posted Date: January 16th, 2021

DOl: https://doi.org/10.21203/rs.3.rs-142994/v1

License: (a) (1) This work is licensed under a Creative Commons Attribution 4.0 International License. Read Full License 


\section{Abstract}

Background: There has been rapid development of migraine research in the past 20 years in China and around the world. We analyzed the development trend of migraine research, and made a bibliometric analysis on the growth trend of the total number of publications, countries, institutions, authors, cited references and keywords from 2000 to 2009 and 2010 to 2019 by applying visualization software, and constructed the relevant knowledge map, and found the trends and hot spots in the field through comparison.

Methods: Retrieved the literature on migraine in the Web of Science database from 2000-2009 and 2010-2019 respectively, and then utilized CiteSpace to conduct bibliometric analysis to acquire the knowledge mapping.

Results: The total number of publications each year has increased year by year, and the average annual growth rate from 2000 to 2009 was $7.999 \%$, while from 2010 to 2019 was $5.348 \%$, with a faster growth rate from 2000 to 2009 . According to the cluster analysis of institutions, authors, cited references, and keywords, 10/11, 12/11, 12/11, and 8/9 categories were obtained from 2000 to 2009 and 2010 to 2019 respectively. The most productive countries, institutions, and authors are the USA, Albert Einstein College of Medicine, and Lipton RB from 2000 to 2009 and 2010 to 2019, whose frequency is 4274/7107, 322/592, and 279/472 respectively. However, the most important of them are Belgium/ Pakistan, Houston Headache Clin/ Amgen Inc, and Lipton RB/ Klatt J, owing to their highest centrality, they are 1.20/1.08, 1.13/1.32, and 1.22/0.89 separately. Moreover, Cited references that contributed to the most co-citations are Silberstein SD (2004)/ Bes A (2013) from 2000 to 2009 and 2010 to 2019, however, the most vital cited reference is Welch KMA (2001)/ Goadsby PJ (2017). Keywords such as migraine, headache, prevalence, and the double blind are the most frequently used. But prevalence is the crucial keyword. In the cluster analysis of institutions, authors, cited references, and keywords from 2000 to 2009 and 2010 to 2019, the more convincing research categories are potential antimigraine agent/ chronic migration prevention, reducing headache recurrence/ following symptomatic treatment, to-left shunt/ placebo controlled phase, spectrum study/ family study hemiplegic migraine, with S values of $0.952 / 0.956,0.985 / 0 / 986$, $0.981 / 0.970$ and $0.985 / 0.982$ respectively. Furthermore, the hotspots in this field are "stimulation", "triptan" from 2000 to 2009 and 2010 to 2019.

Conclusion: Based on the bibliometric analysis in the past 20 years, the knowledge mapping of the country, institution, author, cited reference, and the keyword is gained, which has an important guiding significance for quickly and accurately positioning the trend in this field.

\section{Introduction}

Migraine is a group of recurrent headache disease, showing unilateral or bilateral pain, often accompanied by nausea and vomiting. A few typical cases have visual, sensory, and motor disorders and other auras before the attack[1], and may have a family history[2]. According to a study of the 2016 global burden of disease study, 1.04 billion people worldwide suffer from migraine, with an overall prevalence rate of 14.4\%[3]. Migraine affects women three times as much as men[4]. The prevalence of migraine in children increases with age[5]. And the annual prevalence of migraine in the elderly is about $10 \%[6]$. Migraine is the main cause of disability in the world, the number of years of disability-adjusted life lost caused by migraine has reached one third[7]. With the increase of headache days, the disease burden (disability, medical care utilization, and direct cost) of migraine patients also increases[8]. The high incidence rate, high disability rate, and the increase of treatment expenditure have brought serious economic costs and brought various burdens to society. It is vital to quickly and accurately grasp the research trends of migraine.

Bibliometrics is an interdisciplinary science that uses mathematical and statistical methods to quantitatively analyze all knowledge carriers. It uses statistical indicators to measure the contribution of a research field[9], including different countries, institutions, journals, or authors and predicts trends or hotspots in a field[10]. The research methods involved include co-word analysis and cluster analysis. Co-word analysis is an important method of bibliometrics[11]. It can be used to identify trends and hotspots. The number of occurrences in the group of literature, this number of co-occurrences to measure the close relationship between them. Cluster analysis utilizes weighting algorithm, log-likelihood algorithm, and mutual information algorithm with "title entries", "index entries", and "abstract entries" to extract common information to interpret research mainstreams and research 
characteristics[12]. Researchers rely on relevant analysis software for visual analysis (including Citespace[13], VOSviewer[14], Histcite[15], etc.).

To date, there has been no comprehensive article of studies on migraine using bibliometric methods. Therefore, in this study, to analyze the global status and trend of migraine in the past 20 years, the researchers used the bibliometric software CiteSpace to not only analyze the growth of publications in this field from 2000 to 2019 of the web of Science Database (SCI-E), but also construct the knowledge maps of countries, institutions, authors, cited references and keywords from 2000 to 2009 and 2010 to 2019 , and compare the differences and connections between them, which is of great significance to accurately grasp the research trends and hotspots.

\section{Methods}

\subsection{Source of literature}

To prevent the omission of searching the literature, we used "migraine" to search for synonyms in the MeSH Database, the subject word database in Pubmed, and then merge the final data. Firstly, we input the Web of Science database with English Subject words= "migraine". Next is Web of science database settings: Literature was retrieved online through the Science Citation Index-Expanded of the Web of Science Core Collection on November 18, 2020[16], and the language of the literature is not limited. The search range of the database is 2000-2009 and 2010-2019. Then the analysis is based on this. After literature retrieval, 13194 articles and 20994 articles were obtained in 2000-2009 and 2010-2019 respectively, and 12710 pieces of literature and 20139 literature were used for scientometric analysis after Citespace removed duplication. The elimination of literature duplication was carried out in the form of CiteSpace software and manual verification. First, Citespace software is used to deduplicate literature, secondly, two researchers were required to check before and after literature deduplication. These two researchers separately examined the papers by title, abstract, and full text. And the searched Web of science database comes from the Tsinghua University Library database in China.

\subsection{Analysis software}

The version of Citespace analysis software is 5.2.R1, which was invented by Professor Chen Chaomei from the Computer and Information Science of Drexel University in the United States, and can be used to analyze the structure, laws, and distribution of scientific knowledge[17].

\subsection{Download and import of data}

The results retrieved by subject words are exported, and the file format is "plain text". The researchers used Citespace software to remove duplicates and kept a unique record, and then analyzed it.

\subsection{Parameter setting}

Time slicing (from 2000 to 2009 and from 2010 to 2019, years per slice: 2), node type (check one at a time, including Author, Institution, Country, Keyword, Cited Reference), selection criteria (TopN, select top 50 levels of most cited or occurred items from each slice), pruning select Pathfinder, and Pruning the merged network. Because the network obtained by keywords is messy in 2010-2019, Therefore, the network screening measures of keywords are as follows: years per slice: 2 , the keyword analysis should also select pruning sliced networks.

\subsection{Statistical methods}

The statistics of the total number of publications in the two stages were analyzed by SPSS 24.0, and the measurement data is expressed by the mean \pm standard deviation. If it is following the normal distribution, the comparison of the means between the two groups is performed by $t$-test. Relying on GraphPad Prism 8 to perform linear regression analysis on the total number of publications. And the difference is statistically significant with $P<0.01$. The scientometric analysis was executed on all literature of acupuncture for cancer pain, the frequency of which is mainly utilized to observe the core countries/territories, institutions, authors, cited references, keywords. And Centrality is an indicator to measure the importance of nodes in the network, to discover 
and measure the significance of the literature. The result of clustering analysis is a keyword co-occurrence network. This network can reflect the current and past research hotspots in a certain field. Cluster view shows the distribution of research fields from different angles. Based on the network structure and the clarity of clustering, Citespace provides two indicators: the module value (referred to as $Q$ value) and the silhouette value (referred to as $S$ value), as the basis for us to judge the effect of map drawing, the silhouette value is the average that the contour values of each sample point. Generally speaking, the $Q$ value is generally in the interval $[0,1)$, and $Q>0.3$ means that the divided community structure is significant; when the $S$ value is 0.7 , the clustering is highly efficient and convincing, if it is 0.5 above, clustering is generally considered reasonable[18]. And the interpretation of cluster analysis results mainly includes Cluster ID, Mean Year, Size, Silhouette, Label (LLR), Label (MI). Cluster ID is the number after clustering, and Size represents the number of members contained in the cluster. The larger the Size, the smaller the number. Mean Year represents the average year of the literature in the cluster, which can be used to judge the distance of the cited literature in the cluster. The larger the log-likehood ratio (LLR), the more representative of the cluster category; mutual information (MI) is mainly used to represent the relationship between terms and categories in text mining, and it does not consider the frequency of feature words. The timeline view mainly shows solicitude for delineating the relationship between clustering results and focuses on the historical span of documents in a clustering result.

\section{Results}

\subsection{Analysis of the total number of publications}

The variable of the total number of publications in the two time periods was found to obey a normal distribution through P-P chart analysis. Therefore, the comparison of the two means found that $P=0.000128<0.01$ (Table 1 ), and the difference is statistically significant. The average number of publications increased from 1271.0 in 2000-2009 to 2013.9 in 2010-2019. From the perspective of the average annual growth rate, the growth rate from 2000 to 2009 was $7.999 \%$, which was greater than the $5.348 \%$ from 2010 to 2019, indicating that the total number of migraine publications from 2000 to 2009 increased faster than that from 2010 to 2019, and the total number of publications has also increased from 855 in 2000 to 2,757 in 2019 (Fig. 1), which can also be proved from the linear regression analysis results. The linear regression equation for $2000-2009$ is $Y=102.2 X$ +708.7 (Fig. 1 and Table 1), the Pearson $r$ correlation coefficient $=0.9348, P<0.0001$, indicating that it is greater than $99.5 \%$ Confident that the correlation coefficient reaches $0.9348, X$ and $Y$ are linearly related; while the linear regression equation for 2010-2019 is $Y=101.7 X+1455$ (Fig. 1 and Table 1), the Pearson $r$ correlation coefficient $=0.8713$, and $P=0.001<0.01$, indicating that it is greater than $99.5 \%$ Confident that the correlation coefficient reaches $0.8713, X$ and $Y$ are linearly related.

Table 1

Comparison of the mean that the total number of publications per year in two different periods

\begin{tabular}{|llllll|}
\hline Group & TNP $(\overline{\mathbf{x}} \pm \mathbf{s})$ & The average annual growth rate & $\boldsymbol{F}$ & $\boldsymbol{P}$-Value \\
\hline $2000-2009$ & $1271.0000 \pm 331.15086$ & $7.999 \%$ & 0.007 & 0.000128 \\
\hline $2010-2019$ & $2013.9000 \pm 353.36240$ & $5.348 \%$ & & \\
\hline $\mathrm{TNP}=$ Total Number of Publications & & & \\
\hline $\mathrm{Y}=102.2^{*} \mathrm{X}+708.7$ & & & \\
\hline $\mathrm{Y}=101.7^{*} \mathrm{X}+1455$ & & & \\
\hline
\end{tabular}

\subsection{Distribution of Countries/Territories/Institutions and Cluster analysis}

Comparing the high-yielding countries/territories in the two time periods, it is found that the USA, Italy, Germany, and England are the four countries/territories with the most published migraine studies in the two periods, and the number of papers published in the two periods has been increasing (Table 2). Besides, the number of migraine studies published in China from 2010 to 2019 also jumped to fifth place. However, from the perspective of centrality, the countries/territories engaged in migraine research from 2000 to 2009 are mainly Belgium, Netherlands, Hungary, and their centrality are 1.20, 1.12, and 0.74 respectively; and the most 
important countries from 2010 to 2019 are Pakistan (centrality = 1.08), U Arab Emirates (centrality = 1.08) and Ireland (centrality = 0.88). 
Table 2

Countries/Territories/Institutions contributed to publications on migraine from 2000 to 2009 and 2010 to 2019

\begin{tabular}{|c|c|c|c|c|c|c|c|c|}
\hline $\begin{array}{l}\text { Different } \\
\text { time } \\
\text { periods }\end{array}$ & $\begin{array}{l}\text { Country/ } \\
\text { Territories }\end{array}$ & Frequency & Centrality & $\begin{array}{l}\text { Country/ } \\
\text { Territories }\end{array}$ & Institution & Frequency & Centrality & Institution \\
\hline \multirow[t]{15}{*}{$\begin{array}{l}2000- \\
2009\end{array}$} & USA & 4274 & 1.20 & Belgium & $\begin{array}{l}\text { Albert } \\
\text { Einstein Coll } \\
\text { Med }\end{array}$ & 322 & 1.13 & $\begin{array}{l}\text { Houston } \\
\text { Headache } \\
\text { Clin }\end{array}$ \\
\hline & Italy & 1303 & 1.12 & Netherlands & $\begin{array}{l}\text { Harvard } \\
\text { Univ }\end{array}$ & 278 & 0.73 & $\begin{array}{l}\text { Albert } \\
\text { Einstein Coll } \\
\text { Med }\end{array}$ \\
\hline & Germany & 1198 & 0.74 & Hungary & $\begin{array}{l}\text { Univ Roma } \\
\text { La Sapienza }\end{array}$ & 236 & 0.69 & Mayo Clin \\
\hline & England & 1048 & 0.67 & Poland & $\begin{array}{l}\text { Univ } \\
\text { Copenhagen }\end{array}$ & 213 & 0.69 & Duke Univ \\
\hline & Spain & 522 & 0.65 & England & Leiden Univ & 192 & 0.64 & Leiden Univ \\
\hline & Netherlands & 508 & 0.65 & Croatia & $\begin{array}{l}\text { New } \\
\text { England Ctr } \\
\text { Headache }\end{array}$ & 171 & 0.64 & $\begin{array}{l}\text { Diamond } \\
\text { Headache } \\
\text { Clin Ltd }\end{array}$ \\
\hline & France & 465 & 0.64 & Mexico & Mayo Clin & 152 & 0.57 & $\begin{array}{l}\text { Univ Essen } \\
\text { Gesamthsch }\end{array}$ \\
\hline & Canada & 432 & 0.55 & Ecuador & $\begin{array}{l}\text { Univ Calif } \\
\text { Los Angeles }\end{array}$ & 151 & 0.57 & Lipton \\
\hline & Denmark & 330 & 0.47 & Greece & Inst Neurol & 142 & 0.51 & $\begin{array}{l}\text { Thomas } \\
\text { Jefferson } \\
\text { Univ }\end{array}$ \\
\hline & Turkey & 328 & 0.41 & Bulgaria & $\begin{array}{l}\text { Thomas } \\
\text { Jefferson } \\
\text { Univ }\end{array}$ & 135 & 0.45 & $\begin{array}{l}\text { Ortho } \\
\text { McNeil } \\
\text { Janssen Sci } \\
\text { Affairs LLC }\end{array}$ \\
\hline & Brazil & 297 & 0.30 & Colombia & $\begin{array}{l}\text { UCL Natl } \\
\text { Hosp Neurol } \\
\& \text { Neurosurg }\end{array}$ & 120 & 0.45 & $\begin{array}{l}\text { Univ } \\
\text { Erlangen } \\
\text { Nurnberg }\end{array}$ \\
\hline & Japan & 296 & 0.28 & USA & $\begin{array}{l}\text { Univ Essen } \\
\text { Gesamthsch }\end{array}$ & 109 & 0.39 & $\begin{array}{l}\text { New } \\
\text { England Ctr } \\
\text { Headache }\end{array}$ \\
\hline & Australia & 257 & 0.27 & Chile & $\begin{array}{l}\text { Univ } \\
\text { Cincinnati }\end{array}$ & 106 & 0.39 & $\begin{array}{l}\text { Michigan } \\
\text { Head Pain \& } \\
\text { Neurol Inst }\end{array}$ \\
\hline & Belgium & 242 & 0.20 & Switzerland & Univ Liege & 98 & 0.37 & Drexel Univ \\
\hline & Sweden & 232 & 0.20 & Finland & $\begin{array}{l}\text { Merck \& Co } \\
\text { Inc }\end{array}$ & 95 & 0.36 & $\begin{array}{l}\text { UCL Natl } \\
\text { Hosp Neurol } \\
\& \text { Neurosurg }\end{array}$ \\
\hline \multirow[t]{3}{*}{$\begin{array}{l}2010- \\
2019\end{array}$} & USA & 7107 & 1.08 & Pakistan & $\begin{array}{l}\text { Albert } \\
\text { Einstein Coll } \\
\text { Med }\end{array}$ & 592 & 1.32 & Amgen Inc \\
\hline & Italy & 2382 & 1.08 & $\begin{array}{l}\text { U Arab } \\
\text { Emirates }\end{array}$ & $\begin{array}{l}\text { Univ } \\
\text { Copenhagen }\end{array}$ & 530 & 0.96 & Vedanta Res \\
\hline & Germany & 1516 & 0.88 & Ireland & Mayo Clin & 487 & 0.94 & $\begin{array}{l}\text { Albert } \\
\text { Einstein Coll } \\
\text { Med }\end{array}$ \\
\hline
\end{tabular}

Note: For specific abbreviations, please refer to the abbreviations at the end of the article. 


\begin{tabular}{|c|c|c|c|c|c|c|c|c|}
\hline $\begin{array}{l}\text { Different } \\
\text { time } \\
\text { periods }\end{array}$ & $\begin{array}{l}\text { Country/ } \\
\text { Territories }\end{array}$ & Frequency & Centrality & $\begin{array}{l}\text { Country/ } \\
\text { Territories }\end{array}$ & Institution & Frequency & Centrality & Institution \\
\hline & England & 1493 & 0.65 & Malaysia & $\begin{array}{l}\text { Harvard } \\
\text { Univ }\end{array}$ & 355 & 0.90 & $\begin{array}{l}\text { Montefiore } \\
\text { Med Ctr }\end{array}$ \\
\hline & $\begin{array}{l}\text { Peoples R } \\
\text { China }\end{array}$ & 866 & 0.60 & $\begin{array}{l}\text { Saudi } \\
\text { Arabia }\end{array}$ & Leiden Univ & 334 & 0.82 & $\begin{array}{l}\text { Montefiore } \\
\text { Headache } \\
\text { Ctr }\end{array}$ \\
\hline & Denmark & 832 & 0.53 & Norway & $\begin{array}{l}\text { Kings Coll } \\
\text { London }\end{array}$ & 324 & 0.77 & $\begin{array}{l}\text { Novartis } \\
\text { Pharma AG }\end{array}$ \\
\hline & Spain & 825 & 0.39 & Switzerland & $\begin{array}{l}\text { Univ Calif } \\
\text { San } \\
\text { Francisco }\end{array}$ & 277 & 0.73 & $\begin{array}{l}\text { Sapienza } \\
\text { Univ Rome }\end{array}$ \\
\hline & Turkey & 759 & 0.39 & Estonia & $\begin{array}{l}\text { Harvard } \\
\text { Med Sch }\end{array}$ & 250 & 0.62 & $\begin{array}{l}\text { Sapienza } \\
\text { Univ }\end{array}$ \\
\hline & Netherlands & 716 & 0.28 & Belgium & $\begin{array}{l}\text { Thomas } \\
\text { Jefferson } \\
\text { Univ }\end{array}$ & 243 & 0.58 & Univ Pavia \\
\hline & Canada & 672 & 0.28 & $\begin{array}{l}\text { New } \\
\text { Zealand }\end{array}$ & $\begin{array}{l}\text { Univ Calif } \\
\text { Los Angeles }\end{array}$ & 236 & 0.57 & Charite \\
\hline & France & 630 & 0.25 & Wales & Univ Pavia & 223 & 0.54 & $\begin{array}{l}\text { Harvard } \\
\text { Univ }\end{array}$ \\
\hline & Australia & 531 & 0.18 & USA & $\begin{array}{l}\text { Univ Roma } \\
\text { La Sapienza }\end{array}$ & 205 & 0.50 & $\begin{array}{l}\text { Univ Roma } \\
\text { La Sapienza }\end{array}$ \\
\hline & Brazil & 527 & 0.18 & Netherlands & $\begin{array}{l}\text { Montefiore } \\
\text { Med Ctr }\end{array}$ & 199 & 0.45 & $\begin{array}{l}\text { Univ } \\
\text { Mississippi }\end{array}$ \\
\hline & Norway & 434 & 0.18 & Greece & Amgen Inc & 175 & 0.42 & $\begin{array}{l}\text { Univ } \\
\text { Cincinnati }\end{array}$ \\
\hline & Switzerland & 429 & 0.17 & Scotland & $\begin{array}{l}\text { Univ Sao } \\
\text { Paulo }\end{array}$ & 168 & 0.40 & $\begin{array}{l}\text { Cincinnati } \\
\text { Childrens } \\
\text { Hosp Med } \\
\text { Ctr }\end{array}$ \\
\hline
\end{tabular}

Note: For specific abbreviations, please refer to the abbreviations at the end of the article.

In 2000-2009 and 2010-2019, two network graphs containing 93 nodes and 88 countries/territories or 66 nodes and 73 countries/territories links were generated respectively (Figure 2), and two cluster maps with 117 nodes and 121 links (Modularity $\mathrm{Q}=0.8154$, Silhouette $=0.4925$ ) or with 113 nodes and 115 links (Modularity $Q=0.8261$, Silhouette $=0.6720$ ) of institutions were emerged, along with timeline view (Figure 3). Rely on the cluster analysis of institutions, researchers can find the same research category that different institutions are engaged in. This point is mainly based on the cluster view Figure 3(A) and Table 3. When the cluster view can't judge which institutions focus on the same research in detail, it will utilize the cluster timeline view Figure 3(B) to analyze. The whole modularity $Q=0.8154>0.3$ in 2000-2009, and whole modularity $Q=0.8261>0.3$ in 2010-2019, which indicates that the results of the divided community structure are significant. 10 categories were obtained by cluster analysis in 2000-2009, which were $₫ 0$ akershus study ( $S=0.913>0.7$, owns 16 institutions), $\otimes 1$ comparative trial ( $S=0.949>0.7$, owns 13 institutions), $\otimes 2$ spectrum study ( $\mathrm{S}=0.918>0.7$, owns 13 institutions), $\varangle 3$ acute treatment $(S=0.909>0.7$, owns 12 institutions), $\nabla 4$ naproxen sodium $500 \mathrm{mg}(\mathrm{S}=0.710>0.7$, owns 11 institutions), $\nabla 5$ menstrual migraine $(\mathrm{S}=0.772>0.7$, owns 11 institutions), 86 migraine prophylaxis $(S=0.918>0.7$, owns 9 institutions), 87 auditory evoked cortical potential $(S=0.882>0.7$, owns 8 institutions), $\otimes 8$ potential anti-migraine agent $(S=0.952>0.7$, owns 8 institutions), $\varangle 9$ red ear syndrome $(S=0.941>0.7$, owns 8 institutions), and 11 categories were gotten in 2010-2019, including $₫ 0$ headache care, $\otimes 1$ juvenile patient, $₫ 2$ chronic migraine prevention, $₫ 3$ temporomandibular disorder, $\varangle 4$ patient-reported outcome, $₫ 5$ ampp study, $\varangle 6$ systematic review, $₫ 7$ depressive 
disorder, $₫ 8$ pressure algometry data, $\nabla 9$ chronic migraine and $\nabla 10$ childhood maltreatment. The silhouette of all the clustering results was greater than 0.7 , which indicates that the results of all categories of cluster analysis are very reliable. Also, the most important research categories in the two periods were a potential anti-migraine agent and headache care.

Table 3

Institutions engaged in migraine that Details of knowledge clusters from 2000 to 2009 and 2010 to 2019

\begin{tabular}{|c|c|c|c|c|c|c|}
\hline $\begin{array}{l}\text { Different } \\
\text { periods }\end{array}$ & $\begin{array}{l}\text { Cluster } \\
\text { ID }\end{array}$ & Size & Silhouette & $\begin{array}{l}\text { Mean } \\
\text { (Year) }\end{array}$ & Label (LLR) & Label (MI) \\
\hline \multirow{10}{*}{$2000-2009$} & 0 & 16 & 0.913 & 2003 & akershus study & self-reported muscle tension \\
\hline & 1 & 13 & 0.949 & 2002 & comparative trial & self-reported muscle tension \\
\hline & 2 & 13 & 0.918 & 2003 & spectrum study & self-reported muscle tension \\
\hline & 3 & 12 & 0.909 & 2002 & acute treatment & visual cortex excitability \\
\hline & 4 & 11 & 0.710 & 2003 & naproxen sodium 500 mg & premenstrual syndrome \\
\hline & 5 & 11 & 0.772 & 2002 & menstrual migraine & self-reported muscle tension \\
\hline & 6 & 9 & 0.918 & 2002 & migraine prophylaxis & ventroposteromedial nucleu \\
\hline & 7 & 8 & 0.882 & 2001 & $\begin{array}{l}\text { auditory evoked cortical } \\
\text { potential }\end{array}$ & self-reported muscle tension \\
\hline & 8 & 8 & 0.952 & 2000 & $\begin{array}{l}\text { potential anti-migraine } \\
\text { agent }\end{array}$ & feline brain \\
\hline & 9 & 8 & 0.941 & 2003 & red ear syndrome & plasma level \\
\hline \multirow[t]{11}{*}{$2010-2019$} & 0 & 13 & 0.919 & 2012 & headache care & pediatric patient \\
\hline & 1 & 12 & 0.956 & 2013 & juvenile patient & two-year follow-up point \\
\hline & 2 & 12 & 0.956 & 2014 & $\begin{array}{l}\text { chronic migraine } \\
\text { prevention }\end{array}$ & $\begin{array}{l}\text { prospective multicentre } \\
\text { descriptive study }\end{array}$ \\
\hline & 3 & 11 & 0.936 & 2012 & $\begin{array}{l}\text { temporomandibular } \\
\text { disorder }\end{array}$ & psychiatric comorbidities \\
\hline & 4 & 10 & 0.916 & 2014 & patient-reported outcome & two-year follow-up point \\
\hline & 5 & 9 & 0.842 & 2012 & ampp study & two-year follow-up point \\
\hline & 6 & 9 & 0.805 & 2011 & systematic review & two-year follow-up point \\
\hline & 7 & 9 & 0.969 & 2010 & depressive disorder & two-year follow-up point \\
\hline & 8 & 9 & 0.930 & 2011 & pressure algometry data & $\begin{array}{l}\text { medieval headache } \\
\text { classification }\end{array}$ \\
\hline & 9 & 8 & 0.942 & 2012 & chronic migraine & two-year follow-up point \\
\hline & 10 & 7 & 0.910 & 2012 & childhood maltreatment & brain excitability \\
\hline
\end{tabular}

\subsection{Distribution of authors and Cluster analysis}

The distribution of the main scholars engaged in migraine is shown in Table 4. From 2000 to 2009, the most prolific author was Lipton RB (Frequency = 279), followed by Goadsby PJ (Frequency $=250)$, Diener HC (Frequency $=243$ ), Bigal ME (Frequency = 189), and Silberstein SD (Frequency $=167$ ), the most critical author was also Lipton RB (centrality $=1.22$ ), followed by Dahlof $C$ (centrality = 0.74), O'Quinn S (centrality = 0.74), Cady R (centrality = 0.70) and Diamond M (centrality = 0.67). In 2010-2019, compared with 2000-2009, the most productive author is still Lipton RB (Frequency =472), followed by Goadsby PJ (Frequency = 370), Buse DC (Frequency = 256), Ashina M (Frequency = 238) and Ferrari MD (Frequency = 226), and the most vital author is 
Klatt J (centrality $=0.89)$, followed by Buse DC (centrality $=0.88)$, Mikol DD (centrality $=0.80)$, Hareendran A (centrality $=0.80)$ and Lipton RB (centrality $=0.78$ ), therefore, they are considered to be the most important researcher in the Author's network relationship. 
Table 4

Authors/Cited References contributed to publications on migraine from 2000 to 2009 and 2010 to 2019

\begin{tabular}{|c|c|c|c|c|c|c|c|c|}
\hline \multirow[t]{8}{*}{$\begin{array}{l}\text { Different } \\
\text { periods }\end{array}$} & Author & Frequency & Centrality & Author & $\begin{array}{l}\text { Cited } \\
\text { Reference }\end{array}$ & Frequency & Centrality & $\begin{array}{l}\text { Cited } \\
\text { Reference }\end{array}$ \\
\hline & Lipton RB & 279 & 1.22 & Lipton RB & $\begin{array}{l}\text { Silberstein } \\
\text { SD (2004) }\end{array}$ & 1690 & 1.16 & $\begin{array}{l}\text { Welch KMA } \\
\text { (2001) }\end{array}$ \\
\hline & $\begin{array}{l}\text { Goadsby } \\
\text { PJ }\end{array}$ & 250 & 0.74 & Dahlof C & $\begin{array}{l}\text { Lipton RB } \\
\text { (2001) }\end{array}$ & 543 & 1.11 & $\begin{array}{l}\text { Weiller C } \\
\text { (1995) }\end{array}$ \\
\hline & Diener HC & 243 & 0.74 & O'Quinn S & $\begin{array}{l}\text { Goadsby PJ } \\
(2002)\end{array}$ & 536 & 0.98 & $\begin{array}{l}\text { Solomon } \\
\text { GD (1997) }\end{array}$ \\
\hline & Bigal ME & 189 & 0.70 & Cady R & $\begin{array}{l}\text { Ferrari MD } \\
(2001)\end{array}$ & 322 & 0.95 & $\begin{array}{l}\text { May A } \\
\text { (1998) }\end{array}$ \\
\hline & $\begin{array}{l}\text { Silberstein } \\
\text { SD }\end{array}$ & 167 & 0.67 & $\begin{array}{l}\text { Diamond } \\
\text { M }\end{array}$ & $\begin{array}{l}\text { Ophoff RA } \\
\text { (1996) }\end{array}$ & 300 & 0.95 & $\begin{array}{l}\text { Goadsby PJ } \\
\text { (1993) }\end{array}$ \\
\hline & Ferrari MD & 163 & 0.51 & Massiou H & $\begin{array}{l}\text { Silberstein } \\
\text { SD (2000) }\end{array}$ & 285 & 0.95 & $\begin{array}{l}\text { Goadsby PJ } \\
\text { (1994) }\end{array}$ \\
\hline & Olesen $\mathrm{J}$ & 145 & 0.48 & Diener HC & $\begin{array}{l}\text { Hadjikhani N } \\
(2001)\end{array}$ & 257 & 0.94 & $\begin{array}{l}\text { Rapoport } \\
\text { AM (1997) }\end{array}$ \\
\hline \multirow[t]{15}{*}{$\begin{array}{l}2000- \\
2009\end{array}$} & $\begin{array}{l}\text { Schoenen } \\
\text { J }\end{array}$ & 129 & 0.45 & Dowson AJ & $\begin{array}{l}\text { De FM } \\
(2003)\end{array}$ & 248 & 0.94 & $\begin{array}{l}\text { Longmore J } \\
\text { (1997) }\end{array}$ \\
\hline & $\begin{array}{l}\text { Bussone } \\
\text { G }\end{array}$ & 115 & 0.45 & $\begin{array}{l}\text { MacGregor } \\
\text { EA }\end{array}$ & $\begin{array}{l}\mathrm{Hu} \text { XH } \\
(1999)\end{array}$ & 248 & 0.92 & $\begin{array}{l}\text { Vandenbrink } \\
\text { AM (1998) }\end{array}$ \\
\hline & $\begin{array}{l}\text { Dodick } \\
\text { DW }\end{array}$ & 106 & 0.44 & Reed ML & $\begin{array}{l}\text { Olesen J } \\
(2004)\end{array}$ & 245 & 0.86 & $\begin{array}{l}\text { Ayata C } \\
(2006)\end{array}$ \\
\hline & Nappi G & 105 & 0.36 & Stewart WF & $\begin{array}{l}\text { Kruit MC } \\
(2004)\end{array}$ & 242 & 0.81 & $\begin{array}{l}\text { Pascual J } \\
(2000)\end{array}$ \\
\hline & Evers S & 99 & 0.35 & Lines CR & $\begin{array}{l}\text { Lipton RB } \\
\text { (2001) }\end{array}$ & 242 & 0.78 & $\begin{array}{l}\text { Perry CM } \\
(1998)\end{array}$ \\
\hline & Pascual J & 86 & 0.35 & $\begin{array}{l}\text { McCarroll } \\
\mathrm{KA}\end{array}$ & $\begin{array}{l}\text { Brandes JL } \\
(2004)\end{array}$ & 236 & 0.78 & $\begin{array}{l}\text { Mathew NT } \\
\text { (1997) }\end{array}$ \\
\hline & Tepper SJ & 84 & 0.34 & Kolodner K & $\begin{array}{l}\text { Tfelt-hansen } \\
\text { P (2000) }\end{array}$ & 231 & 0.59 & $\begin{array}{l}\text { Scher Al } \\
(2003)\end{array}$ \\
\hline & $\begin{array}{l}\text { Rapoport } \\
\text { AM }\end{array}$ & 75 & 0.33 & Lipton R & $\begin{array}{l}\text { Bolay H } \\
(2002)\end{array}$ & 224 & 0.57 & $\begin{array}{l}\text { Hadjikhani } \\
N(2001)\end{array}$ \\
\hline & Lipton RB & 472 & 0.89 & Klatt J & Bes A (2013) & 1711 & 0.89 & $\begin{array}{l}\text { Goadsby PJ } \\
\text { (2017) }\end{array}$ \\
\hline & $\begin{array}{l}\text { Goadsby } \\
\text { PJ }\end{array}$ & 370 & 0.88 & Buse DC & $\begin{array}{l}\text { Silberstein } \\
\text { SD (2004) }\end{array}$ & 1307 & 0.75 & $\begin{array}{l}\text { Dodick DW } \\
\text { (2014) }\end{array}$ \\
\hline & Buse DC & 256 & 0.80 & Mikol DD & $\begin{array}{l}\text { Stovner LJ } \\
\text { (2007) }\end{array}$ & 481 & 0.73 & $\begin{array}{l}\text { Silberstein } \\
\text { SD (2012) }\end{array}$ \\
\hline & Ashina M & 238 & 0.80 & $\begin{array}{l}\text { Hareendran } \\
\text { A }\end{array}$ & $\begin{array}{l}\text { Lipton RB } \\
\text { (2007) }\end{array}$ & 467 & 0.71 & $\begin{array}{l}\text { Tepper S } \\
(2017)\end{array}$ \\
\hline & Ferrari MD & 226 & 0.78 & Lipton RB & $\begin{array}{l}\text { Olesen J } \\
(2006)\end{array}$ & 384 & 0.71 & $\begin{array}{l}\text { Hepp Z } \\
\text { (2015) }\end{array}$ \\
\hline & Olesen J & 217 & 0.78 & Turkel CC & Vos T (2012) & 335 & 0.70 & $\begin{array}{l}\text { Sun H } \\
(2016)\end{array}$ \\
\hline & $\begin{array}{l}\text { Dodick } \\
\text { DW }\end{array}$ & 207 & 0.77 & Zhang F & $\begin{array}{l}\text { Silberstein } \\
\text { SD (2012) }\end{array}$ & 283 & 0.69 & $\begin{array}{l}\text { Lipton RB } \\
\text { (2007) }\end{array}$ \\
\hline
\end{tabular}




\begin{tabular}{|c|c|c|c|c|c|c|c|c|}
\hline $\begin{array}{l}\text { Different } \\
\text { periods }\end{array}$ & Author & Frequency & Centrality & Author & $\begin{array}{l}\text { Cited } \\
\text { Reference }\end{array}$ & Frequency & Centrality & $\begin{array}{l}\text { Cited } \\
\text { Reference }\end{array}$ \\
\hline \multirow[t]{8}{*}{$\begin{array}{l}2010- \\
2019\end{array}$} & $\begin{array}{l}\text { Silberstein } \\
\text { SD }\end{array}$ & 182 & 0.73 & Manack A & $\begin{array}{l}\text { Diener HC } \\
(2010)\end{array}$ & 283 & 0.69 & $\begin{array}{l}\text { Ho TW } \\
(2008)\end{array}$ \\
\hline & Diener HC & 180 & 0.71 & DeGryse RE & $\begin{array}{l}\text { Schurks M } \\
\text { (2009) }\end{array}$ & 261 & 0.64 & $\begin{array}{l}\text { Ho TW } \\
(2010)\end{array}$ \\
\hline & $\begin{array}{l}\text { Tassorelli } \\
\text { C }\end{array}$ & 163 & 0.66 & $\begin{array}{l}\text { Lanteri- } \\
\text { Minet M }\end{array}$ & $\begin{array}{l}\text { Aurora SK } \\
(2010)\end{array}$ & 257 & 0.64 & $\begin{array}{l}\text { Munakata J } \\
(2009)\end{array}$ \\
\hline & $\begin{array}{l}\text { Martelletti } \\
\text { P }\end{array}$ & 156 & 0.64 & Barbanti P & $\begin{array}{l}\text { Dodick DW } \\
(2010)\end{array}$ & 247 & 0.57 & $\begin{array}{l}\text { Natoli JL } \\
(2010)\end{array}$ \\
\hline & Wang SJ & 131 & 0.62 & $\begin{array}{l}\text { Martelletti } \\
P\end{array}$ & $\begin{array}{l}\text { Evers S } \\
(2009)\end{array}$ & 242 & 0.52 & $\begin{array}{l}\text { Ho TW } \\
(2008)\end{array}$ \\
\hline & $\begin{array}{l}\text { Terwindt } \\
\text { GM }\end{array}$ & 129 & 0.49 & Diener HC & $\begin{array}{l}\text { Pietrobon D } \\
(2013)\end{array}$ & 240 & 0.49 & $\begin{array}{l}\text { Olesen J } \\
(2009)\end{array}$ \\
\hline & $\begin{array}{l}\text { Schoenen } \\
\text { J }\end{array}$ & 116 & 0.39 & Tassorelli C & $\begin{array}{l}\text { Akerman S } \\
\text { (2011) }\end{array}$ & 238 & 0.46 & $\begin{array}{l}\text { Aurora SK } \\
(2011)\end{array}$ \\
\hline & Grazzi L & 112 & 0.39 & Nappi G & $\begin{array}{l}\text { Blumenfeld } \\
\text { AM (2011) }\end{array}$ & 220 & 0.46 & $\begin{array}{l}\text { Zhang XC } \\
(2011)\end{array}$ \\
\hline
\end{tabular}

2 cluster maps with 142 nodes and 145 links (Fig. $4(A)$, Modularity $Q=0.8357$, Silhouette $=0.5962$ ) or with 140 nodes and 135 links (Fig. $4(C)$, Modularity $Q=0.8469$, Silhouette $=0.6471$ ) of authors were emerged by Citespace, along with timeline view (Fig. 4(B) and Fig. 4(D)). The modularity $Q>0.3$, which indicates that the results of the divided community structure are prominent. Then through the cluster analysis, the researcher can quickly locate the same research content and the relationship between different scholars. The results of cluster analysis are shown in Table 5. During 2000 to 2009, the content of cluster analysis included 12 categories, mainly including \#0 population-based survey ( $S=0.919>0.7$, Contains 17 authors), \#1 menstrual migraine ( $S=0.795>0.7$, Contains 17 authors), \#2 migraine prevention ( $S=0.909>0.7$, Contains 14 authors), \#3 migraine patient $(S=0.957>0.7$, Contains 13 authors), \#4 disintegrating tablet ( $S=0.934>0.7$, Contains 12 authors), \#5 strong point ( $\mathrm{S}=0.973>0.7$, Contains 12 authors), \#6 medication overuse ( $\mathrm{S}=0.812>0.7$, Contains 10 authors), \#7 cardiovascular disease ( $S=0.976>0.7$, Contains 9 authors), \#8 chronic daily headache ( $S=0.978>0.7$, Contains 8 authors), \#9 human isolated blood vessel ( $S=0.926>0.7$, Contains 7 authors), $\# 10$ alternating hemiplegia ( $S=0.953>0.7$, Contains 5 authors), \#11 reducing headache recurrence $(S=0.985>0.7$, Contains 4 authors). In 2010-2019, a total of 11 cluster analysis results were obtained, namely chronic migraine, following symptomatic treatment, patient-reported outcome, eurolight questionnaire, American migraine prevalence, preempt clinical program, medication-overuse headache, genome-wide association study, America symptom, chronic migraine, population-based study. The silhouette of all the clustering results was greater than 0.7 , which indicates that the results of all categories of cluster analysis are very credible. In addition, the most important categories in the two periods were reducing headache recurrence and following symptomatic treatment. 
Table 5

Authors engaged in migraine that Details of knowledge clusters from 2000 to 2009 and 2010 to 2019

\begin{tabular}{|c|c|c|c|c|c|c|}
\hline \multirow[t]{6}{*}{$\begin{array}{l}\text { Different } \\
\text { periods }\end{array}$} & $\begin{array}{l}\text { Cluster } \\
\text { ID }\end{array}$ & Size & Silhouette & $\begin{array}{l}\text { Mean } \\
\text { (Year) }\end{array}$ & Label (LLR) & Label (MI) \\
\hline & 0 & 17 & 0.919 & 2003 & population-based survey & $\begin{array}{l}\text { identifying functional } \\
\text { disability }\end{array}$ \\
\hline & 1 & 17 & 0.795 & 2001 & menstrual migraine & causing cadasil \\
\hline & 2 & 14 & 0.909 & 2002 & migraine prevention & post-marketing experience \\
\hline & 3 & 13 & 0.957 & 2002 & migraine patient & $\begin{array}{l}\text { identifying functional } \\
\text { disability }\end{array}$ \\
\hline & 4 & 12 & 0.934 & 2003 & disintegrating tablet & $\begin{array}{l}\text { identifying functional } \\
\text { disability }\end{array}$ \\
\hline \multirow[t]{11}{*}{$2000-2009$} & 5 & 12 & 0.973 & 2004 & strong point & mtdna a3243g mutation \\
\hline & 6 & 10 & 0.812 & 2002 & medication overuse & $\begin{array}{l}\text { identifying functional } \\
\text { disability }\end{array}$ \\
\hline & 7 & 9 & 0.976 & 2003 & cardiovascular disease & $\begin{array}{l}\text { identifying functional } \\
\text { disability }\end{array}$ \\
\hline & 8 & 8 & 0.978 & 2002 & chronic daily headache & $\begin{array}{l}\text { identifying functional } \\
\text { disability }\end{array}$ \\
\hline & 9 & 7 & 0.926 & 2001 & human isolated blood vessel & $\begin{array}{l}\text { identifying functional } \\
\text { disability }\end{array}$ \\
\hline & 10 & 5 & 0.953 & 2003 & alternating hemiplegia & genetic relation \\
\hline & 11 & 4 & 0.985 & 2004 & $\begin{array}{l}\text { reducing headache } \\
\text { recurrence }\end{array}$ & $\begin{array}{l}\text { functional assessment } \\
\text { measure }\end{array}$ \\
\hline & 0 & 13 & 0.956 & 2014 & chronic migraine & adolescent perspective \\
\hline & 1 & 13 & 0.986 & 2012 & $\begin{array}{l}\text { following symptomatic } \\
\text { treatment }\end{array}$ & adolescent perspective \\
\hline & 2 & 12 & 0.858 & 2015 & patient-reported outcome & adolescent perspective \\
\hline & 3 & 12 & 0.944 & 2011 & eurolight questionnaire & adolescent perspective \\
\hline \multirow[t]{7}{*}{$2010-2019$} & 4 & 12 & 0.805 & 2014 & $\begin{array}{l}\text { american migraine } \\
\text { prevalence }\end{array}$ & adolescent perspective \\
\hline & 5 & 11 & 0.859 & 2011 & preempt clinical program & adolescent perspective \\
\hline & 6 & 11 & 0.966 & 2011 & $\begin{array}{l}\text { medication-overuse } \\
\text { headache }\end{array}$ & adolescent perspective \\
\hline & 7 & 11 & 0.944 & 2012 & $\begin{array}{l}\text { genome-wide association } \\
\text { study }\end{array}$ & adolescent perspective \\
\hline & 8 & 10 & 0.970 & 2013 & america symptom & adolescent perspective \\
\hline & 9 & 10 & 0.979 & 2012 & chronic migraine & adolescent perspective \\
\hline & 10 & 8 & 0.969 & 2011 & population-based study & adolescent perspective \\
\hline
\end{tabular}

\subsection{Distribution of Cited reference and Cluster analysis}

The knowledge base and research progress of migraine are described using high co-citation references and main article clusters respectively, and the co-citation frequencies of references and their importance in network nodes are shown in Table 4. The 
reference with the highest co-citations in 2000-2009 is Silberstein SD (2004)[19] ( Frequency = 1690), followed by Lipton RB (2001) ( Frequency = 543), Goadsby PJ (2002) ( Frequency = 536), Ferrari MD (2001) ( Frequency = 322) and Ophoff RA (1996) ( Frequency $=300$ ), and the highest centrality is Welch KMA (2001)[20] ( Centrality $=1.16$ ) and is regarded as the most important reference in the field, followed by Weiller C (1995) (Centrality = 1.11), Solomon GD (1997) (Centrality = 0.98), May A (1998) ( Centrality $=0.95$ ) and Goadsby PJ (1993) (Centrality = 0.95). Between 2010 and 2019, the reference with the highest co-citations is Bes A (2013) [21] ( Frequency $=1711)$, followed by Silberstein SD (2004) ( Frequency $=1307)$, Stovner LJ (2007) ( Frequency = 481), Lipton RB (2007) (Frequency $=467$ ) and Olesen J (2006) ( Frequency $=384$ ), and the highest centrality is Goadsby PJ (2017)[22] ( Centrality $=0.89$ ), and is considered as the most momentous reference in the field, followed by Weiller C (1995) ( Centrality $=1.11)$, Dodick DW (2014) (Centrality = 0.75), Silberstein SD (2012) (Centrality = 0.73) and Tepper S (2017) (Centrality $=0.71$.

Two cluster maps with 149 nodes and 159 links (Fig. 5(A), Modularity Q $=0.8481$, Silhouette $=0.7417$ ) or with 143 nodes and 141 links (Fig. $5(C)$, Modularity $Q=0.8467$, Silhouette $=0.4431$ ) of references was generated by Citespace, along with timeline view (Fig. 5(B) and Fig. 5(D)). For the cluster analysis (Table 6) between 2000 and 2009, the twelve largest clusters (small clusters were automatically filtered), included \#0 comparative review, \#1 chronic migraine, \#2 economic outcome, \#3 delayed treatment, \#4 contrasting patient preference, \#5 headache sufferers study, \#6 migraine headache, \#7 oral contraceptive, \#8 chronic daily headache, \#9 trigeminovascular system, \#10 using rizatriptan, \#11 to-left shunt. The silhouette of all the clustering results was greater than 0.7, which indicates that all clustering results are dependable. Moreover, the category with the highest silhouette value is \#11 to-left shunt, which shows that the category is the most convincing in this cluster analysis. From 2010 to 2019,11 cluster analysis results were acquired, which were \#0 placebo-controlled phase ( $S=0.970>0.7$, possesses 14 references), \#1 excitatory-inhibitory balance ( $S=0.899>0.7$, possesses 12 references), \#2 chronic migraine $(S=0.947>0.7$, possesses 12 references), \#3 new analysis ( $S=0.955>0.7$, possesses 12 references), \#4 familial hemiplegic migraine $(S=0.966>0.7$, possesses 11 references), \#5 placebo effect $(S=0.909>0.7$, possesses 11 references), \#6 emerging drug $(S=0.904>0.7$, possesses 11 references), \#7 high frequency migraine ( $S=0.957>0.7$, possesses 11 references), \#8 calcium channel function ( $S$ $=0.848>0.7$, possesses 10 references $)$, \#9 key player neuropeptide $(S=0.827>0.7$, possesses 10 references $)$, \#11 familial migraine ( $S=0.895>0.7$, possesses 7 references). All the clustering silhouettes are greater than 0.7 , indicating that the results are very reliable. Furthermore, the category with the highest silhouette value is \#0 placebo-controlled phase, which prompts that the category is the most persuasive in this cluster analysis. 
Table 6

Cited reference engaged in migraine that Details of knowledge clusters from 2000 to 2009 and 2010 to 2019

\begin{tabular}{|c|c|c|c|c|c|c|}
\hline $\begin{array}{l}\text { Different } \\
\text { periods }\end{array}$ & $\begin{array}{l}\text { Cluster } \\
\text { ID }\end{array}$ & Size & Silhouette & $\begin{array}{l}\text { Mean } \\
\text { (Year) }\end{array}$ & Label (LLR) & Label (MI) \\
\hline & 0 & 15 & 0.911 & 1997 & comparative review & migraine \\
\hline & 1 & 14 & 0.979 & 2004 & chronic migraine & migraine \\
\hline & 2 & 14 & 0.912 & 1996 & economic outcome & migraine \\
\hline & 3 & 13 & 0.974 & 1999 & delayed treatment & migraine \\
\hline & 4 & 13 & 0.876 & 2001 & $\begin{array}{l}\text { contrasting patient } \\
\text { preference }\end{array}$ & migraine \\
\hline \multirow[t]{12}{*}{$2000-2009$} & 5 & 12 & 0.976 & 2001 & headache sufferers study & migraine \\
\hline & 6 & 12 & 0.892 & 1997 & migraine headache & calcium channelopathies \\
\hline & 7 & 12 & 0.962 & 2002 & oral contraceptive & migraine \\
\hline & 8 & 12 & 0.979 & 2000 & chronic daily headache & migraine \\
\hline & 9 & 11 & 0.813 & 2003 & trigeminovascular system & underlying mechanism \\
\hline & 10 & 10 & 0.869 & 1995 & using rizatriptan & migraine \\
\hline & 11 & 8 & 0.981 & 2002 & to-left shunt & migraine \\
\hline & 0 & 14 & 0.970 & 2010 & placebo-controlled phase & $\begin{array}{l}\text { to-resting glucose uptake } \\
\text { ratio }\end{array}$ \\
\hline & 1 & 12 & 0.899 & 2009 & $\begin{array}{l}\text { excitatory-inhibitory } \\
\text { balance }\end{array}$ & $\begin{array}{l}\text { to-resting glucose uptake } \\
\text { ratio }\end{array}$ \\
\hline & 2 & 12 & 0.947 & 2010 & chronic migraine & $\begin{array}{l}\text { to-resting glucose uptake } \\
\text { ratio }\end{array}$ \\
\hline & 3 & 12 & 0.955 & 2010 & new analysis & $\begin{array}{l}\text { to-resting glucose uptake } \\
\text { ratio }\end{array}$ \\
\hline & 4 & 11 & 0.966 & 2005 & $\begin{array}{l}\text { familial hemiplegic } \\
\text { migraine }\end{array}$ & $\begin{array}{l}\text { to-resting glucose uptake } \\
\text { ratio }\end{array}$ \\
\hline \multirow[t]{6}{*}{$2010-2019$} & 5 & 11 & 0.909 & 2007 & placebo effect & $\begin{array}{l}\text { to-resting glucose uptake } \\
\text { ratio }\end{array}$ \\
\hline & 6 & 11 & 0.904 & 2016 & emerging drug & $\begin{array}{l}\text { to-resting glucose uptake } \\
\text { ratio }\end{array}$ \\
\hline & 7 & 11 & 0.957 & 2012 & high frequency migraine & $\begin{array}{l}\text { to-resting glucose uptake } \\
\text { ratio }\end{array}$ \\
\hline & 8 & 10 & 0.848 & 2009 & calcium channel function & facial expression \\
\hline & 9 & 10 & 0.827 & 2014 & key player neuropeptide & $\begin{array}{l}\text { pharmacologic } \\
\text { characterization }\end{array}$ \\
\hline & 11 & 7 & 0.895 & 2007 & familial migraine & $\begin{array}{l}\text { to-resting glucose uptake } \\
\text { ratio }\end{array}$ \\
\hline
\end{tabular}

\subsection{Distribution of Keywords and Cluster analysis}

Table 7 shows keywords contributed to publications on migraine, the top five keywords in 2000-2009 are migraine (Frequency = 5308), headache (2413), prevalence (1223), double blind (844), and sumatriptan (754). But from the perspective of centrality, the most vital keyword in the network is migraine (centrality $=1.45$ ), followed by sumatriptan (centrality $=0.97)$, prevalence $(0.96)$, 
headache $(0.85)$, aura (centrality $=0.65)$. Thus, compared with the first stage, migraine, headache, prevalence, double blind were also in the top four, only increasing in number. And the most critical keywords are the same as the previous period, which is migraine (centrality $=1.56$ ). The next four different keywords are double blind (centrality $=1.07)$, prevalence $($ centrality $=0.71)$, aura (centrality $=0.42$ ), and gene related peptide (centrality $=0.38$ ). Therefore, these keywords constitute the main content of migraine research. 
Table 7

Keyword contributed to publications on migraine from 2000 to 2009 and 2010 to 2019

\begin{tabular}{|c|c|c|c|c|}
\hline \multirow[t]{8}{*}{ Different periods } & Keyword & Frequency & Centrality & Keyword \\
\hline & migraine & 5308 & 1.45 & migraine \\
\hline & headache & 2413 & 0.97 & sumatriptan \\
\hline & prevalence & 1223 & 0.96 & prevalence \\
\hline & double blind & 844 & 0.85 & headache \\
\hline & sumatriptan & 754 & 0.65 & aura \\
\hline & pain & 641 & 0.61 & rizatriptan \\
\hline & aura & 564 & 0.58 & efficacy \\
\hline \multirow[t]{15}{*}{$2000-2009$} & epidemiology & 534 & 0.39 & united states \\
\hline & efficacy & 500 & 0.35 & serotonin \\
\hline & population & 479 & 0.29 & patent foramen ovale \\
\hline & children & 461 & 0.25 & stroke \\
\hline & tension type headache & 454 & 0.20 & mutation \\
\hline & united states & 435 & 0.20 & risk factor \\
\hline & familial hemiplegic migraine & 434 & 0.20 & burden \\
\hline & stroke & 400 & 0.15 & familial hemiplegic migraine \\
\hline & migraine & 7831 & 1.56 & migraine \\
\hline & headache & 3903 & 1.07 & double blind \\
\hline & prevalence & 2453 & 0.71 & prevalence \\
\hline & double blind & 1467 & 0.42 & aura \\
\hline & pain & 1413 & 0.38 & gene related peptide \\
\hline & epidemiology & 881 & 0.32 & patent foramen ovale \\
\hline & children & 870 & 0.28 & burden \\
\hline \multirow[t]{8}{*}{$2010-2019$} & chronic migraine & 858 & 0.28 & stroke \\
\hline & aura & 802 & 0.22 & placebo controlled phase \\
\hline & population & 746 & 0.17 & pain \\
\hline & efficacy & 734 & 0.17 & depression \\
\hline & depression & 727 & 0.17 & disability \\
\hline & burden & 700 & 0.17 & cortical spreading depression \\
\hline & tension type headache & 664 & 0.17 & topiramate \\
\hline & quality of life & 648 & 0.17 & onabotulinumtoxina \\
\hline
\end{tabular}

Figure 6 and Table 8 exhibit keyword co-occurrence cluster analysis, 2 knowledge mapping with 76 nodes and 84 keywords links (modular Q $=0.7884$, Mean silhouette $=0.8275$ ) or with 69 nodes and 74 keywords links (modular $\mathrm{Q}=0.7791$, Mean silhouette $=$ 0.8579 ) and cluster view are created. The modularity $Q>0.3$, which indicates that the results of the divided community structure 
are remarkable. The timeline view shows that new keywords appear almost every year. In the cluster map Fig. 6(A), the eight largest clusters (small clusters were automatically filtered) between 2000 and 2009 , contained \#0 spectrum study ( $\mathrm{S}=0.985>$ 0.7 , contains 13 keywords), \#1 trigeminal ganglion cell ( $S=0.917>0.7$, contains 12 keywords), \#2 common experience ( $S=$ $0.850>0.7$, contains 11 keywords), \#3 cytokine level ( $S=0.962>0.7$, contains 11 keywords), \#4 sumatriptan tablet ( $S=0.965>$ 0.7 , contains 9 keywords), \#5 familial hemiplegic migraine ( $S=0.925>0.7$, contains 8 keywords), \#6 to-left shunt $(S=0.905>0.7$, contains 7 keywords), \#7 chronic migraine ( $S=0.939>0.7$, contains 4 keywords). By 2010-2019, cluster analysis has obtained nine research categories, which are \#0 chronic migraine $(S=0.962>0.7$, contains 12 keywords), \#1 chronic migraine $(S=0.946>$ 0.7 , contains 11 keywords), \#2 tension-type headache ( $\mathrm{S}=0.962>0.7$, contains 9 keywords), \#3 transient global amnesia ( $\mathrm{S}=$ $0.936>0.7$, contains 9 keywords), \#4 familial hemiplegic migraine ( $S=0.982>0.7$, contains 8 keywords), \#5 migraine education ( $S=0.955>0.7$, contains 6 keywords), \#6 chronic migraine $(S=0.925>0.7$, contains 5 keywords), \#7 laser-evoked potential ( $S=$ $0.936>0.7$, contains 4 keywords), \#8 anxiety symptom ( $S=0.974>0.7$, contains 4 keywords). The silhouette of all the clustering results was greater than 0.7 , it states clearly that all clustering results are authentic. Hence, after comparing the silhouette values of cluster analysis, it is found that spectrum study and familial hemiplegic migraine are the most important research contents.

Table 8

Keywords engaged in migraine that Details of knowledge clusters from 2000 to 2009 and 2010 to 2019

\begin{tabular}{|c|c|c|c|c|c|c|}
\hline $\begin{array}{l}\text { Different } \\
\text { periods }\end{array}$ & $\begin{array}{l}\text { Cluster } \\
\text { ID }\end{array}$ & Size & Silhouette & $\begin{array}{l}\text { Mean } \\
\text { (Year) }\end{array}$ & Label (LLR) & Label (MI) \\
\hline & 0 & 13 & 0.985 & 2002 & spectrum study & $\begin{array}{l}\text { prothrombotic risk } \\
\text { factor }\end{array}$ \\
\hline & 1 & 12 & 0.917 & 2000 & trigeminal ganglion cell & $\begin{array}{l}\text { prothrombotic risk } \\
\text { factor }\end{array}$ \\
\hline & 2 & 11 & 0.850 & 2001 & common experience & $\begin{array}{l}\text { prothrombotic risk } \\
\text { factor }\end{array}$ \\
\hline \multirow[t]{9}{*}{$2000-2009$} & 3 & 11 & 0.962 & 2000 & cytokine level & $\begin{array}{l}\text { prothrombotic risk } \\
\text { factor }\end{array}$ \\
\hline & 4 & 9 & 0.965 & 2000 & sumatriptan tablet & $\begin{array}{l}\text { prothrombotic risk } \\
\text { factor }\end{array}$ \\
\hline & 5 & 8 & 0.925 & 2002 & $\begin{array}{l}\text { familial hemiplegic } \\
\text { migraine }\end{array}$ & $\begin{array}{l}\text { prothrombotic risk } \\
\text { factor }\end{array}$ \\
\hline & 6 & 7 & 0.905 & 2002 & to-left shunt & $\begin{array}{l}\text { prothrombotic risk } \\
\text { factor }\end{array}$ \\
\hline & 7 & 4 & 0.939 & 2004 & chronic migraine & $\begin{array}{l}\text { prothrombotic risk } \\
\text { factor }\end{array}$ \\
\hline & 0 & 12 & 0.962 & 2011 & chronic migraine & occipital nerve block \\
\hline & 1 & 11 & 0.946 & 2011 & chronic migraine & occipital nerve block \\
\hline & 2 & 9 & 0.962 & 2010 & tension-type headache & occipital nerve block \\
\hline & 3 & 9 & 0.936 & 2010 & transient global amnesia & occipital nerve block \\
\hline \multirow[t]{5}{*}{ 2010-2019 } & 4 & 8 & 0.982 & 2011 & $\begin{array}{l}\text { familial hemiplegic } \\
\text { migraine }\end{array}$ & triggering migraine \\
\hline & 5 & 6 & 0.955 & 2010 & migraine education & occipital nerve block \\
\hline & 6 & 5 & 0.925 & 2012 & chronic migraine & occipital nerve block \\
\hline & 7 & 4 & 0.936 & 2010 & laser-evoked potential & occipital nerve block \\
\hline & 8 & 4 & 0.974 & 2010 & anxiety symptom & alcohol dependence \\
\hline
\end{tabular}


Burst is used to reflecting hotspots within a certain time node. The red bar indicates the duration of the citation explosion and the start and end year that the citation explosion. That is to say: the red bar indicates some frequently cited keywords, and the green bar is rarely cited in terms of keywords[23], it was found that the hotspot of migraine between 2000 and 2009 was "stimulation". And from 2010 to 2019, the hotspot of migraine was "triptan". The specific results are shown in Fig. 7.

\section{Discussions}

In our studies, we completed a scientometric analysis of migraine research from 2000 to 2019 and constructed a knowledge map of countries/territories, institutions, authors, keywords, and Cited references. After analyzing the development trend of the total number of publications, we found an interesting phenomenon: in the past 20 years, the overall development trend of the total number of migraine research publications has been increasing year by year, and the average total number of publications from 2010 to 2019 (2013.9) is greater than 2000-2009 (1271.0), but the average growth rate for 2000-2009 (7.999\%) is greater than 2010-2019 (5.348\%), and the slope of the linear regression equation is also compared with 2000-2009. The slope (102.2) is greater than 2010-2019 (101.7), indicating that publications in 2000-2009 grew faster. Like its economic power, Developed countries, led by the United States, occupies an important position in migraine research, not only in high output, but also in high contribution, but from the analysis of the importance of national cooperation networks, by 2010-2019, South Asia (Pakistan), Southeast Asia (Malaysia), and the Middle East (UAE, Saudi Arabia) have developed rapidly, and they play a more prominent role in communicating cooperation between countries. Besides, China in East Asia has increased more rapidly in the total number of publications and has jumped to the top five. These developments are very momentous, but developed countries still dominate migraine research. It can also be confirmed by the analysis of institutions. In two decades, the Albert Einstein College of Medicine has published the most migraine research. In the analysis of the importance of the cooperation network, Houston Headache Clinic from 2000 to 2009 and Amgen Inc from 2010 to 2019 are from the USA. Global migraine research has achieved amazing results, increasing from 855 papers in 2000 to 2,757 in 2019. These have brought a lot of great encouragement to those who suffer from migraines.

\section{Authors' cluster analysis comparison}

By comparing the cluster analysis of the authors in the two time periods, it is found that the common studies are based on population survey, while the studies in 2000-2009 mainly focus on migraine in the special population[24], migraine epidemiology and health care model in the United States[25], and migraine prevalence in Taipei (China) [26]. And the 2010-2019 study is more inclined to the treatment of children's migraine[27], chronic pain, and depression in the quality of life of migraine women[28]. Cluster analysis of authors from 2000 to 2009: $\nabla \# 1$ menstrual migraine: First, the relationship between the menstrual cycle and headaches of migraine patients were explored, and it was confirmed that migraine attacks without aura were more likely to occur 2 days before the start of menstruation and 2 days before the menstrual cycle[29]. Secondly, it was found that rizatriptan can effectively treat menstrual-related migraine attacks[30]. All these provide a good research basis for the diagnosis and treatment

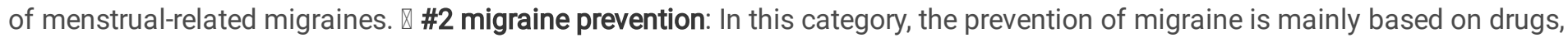
and the representative drug is naratriptan. Related results indicate that if $2.5 \mathrm{mg}$ of naratriptan is taken in the early stage of prodromal symptoms, it is more effective to prevent headaches and the severity of headaches will be greatly reduced, which provides a medication basis for the early prevention of migraine[31]. ॠ\#3migraine patient: Through the comparative analysis of visual and auditory cortex evoked potentials in migraine patients, it is found that the increased migraine response may be due to the decreased pre-activation level of the sensory cortex[32].囚\#4 disintegrating tablet: A Phase 3 crossover study on the pharmacokinetic characteristics of $10 \mathrm{mg}$ rizatriptan tablets and orally disintegrating tablets in healthy subjects proved that the absorption rate of rizatriptan orally disintegrating tablets was slightly slower than that of its tablets, and the bioavailability and peak concentration were similar[33], which supplied a reference method for the study of the absorption rate on different dosage forms of drugs. $\mathbb{\square}$ \# medication overuse: This category engrosses in the follow-up study of overuse of analgesics in the treatment of migraine. In the follow-up study after 3 years of overuse of analgesics, the combination of drugs and biofeedback therapy is more effective than drug therapy alone[34], which provides a new treatment model for migraine. Overuse of analgesics

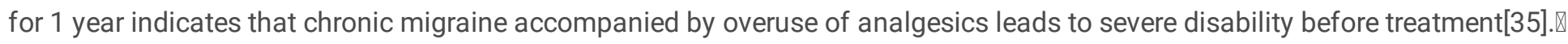

\#7 cardiovascular disease: This category mainly discusses the relationship between migraine and cardiovascular disease. Studies have shown that TT genotype carriers have an increased risk of cardiovascular disease in migraine patients with 
aura[36], while researches in female migraine patients have found that active migraine with aura is associated with an increased risk of cardiovascular disease, active migraine without aura is not associated with an increased risk of cardiovascular events[37]. This is a good exploration from the correlation research of risk factors. $\mathbb{Z}$ \# chronic daily headache: The research in this category mainly includes two aspects of the main life changes before and after the onset of chronic daily headache and the identification of chronic daily headache and related factors of induction and transformation. A proportional control study found that chronic daily headache cases are more likely to experience certain major life events within 2 years before the chronic daily headache attack than episodic headaches, especially in middle-aged people over 40 years[38]. In addition, the transformation of past occasional headaches or the occurrence of new daily persistent headaches may be related to certain medical conditions and behaviors[39], providing a basis for the analysis of related factors of induction and transformation. $\mathbb{\nabla} \mathbf{\#}$ human isolated blood vessel: Eletriptan and sumatriptan contract the middle meningeal artery more than the coronary artery[40], which provides a basis

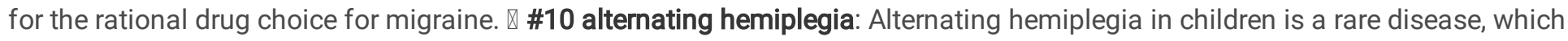
is often associated with migraine. CACNA1A gene on chromosome 19 is related to familial hemiplegic migraine and other paroxysmal brain diseases. The mutation of the CANA1A gene is expected to increase the concentration of $\mathrm{K}+$ and glutamate in synaptic space, and increase the release of neurotransmitters, which is the mechanism of alternating hemiplegia and migraine in children[41]. Haan J[42] found that the second comorbid gene is ATP1A2, which is the common pathogenesis of alternating hemiplegia and migraine in children. Cluster analysis of authors from 2010 to 2019: $\triangle$ Chronic migraine: \#0 and \#9 are included in this category, Oral botulinum toxin not only significantly reduced the number of days of headache and migraine but also improved the quality of life for patients and avoided the overuse of painkillers[43]. \\#2patient-reported outcome: Focusing on patient outcomes in migraine prevention, Pompili M's study[44] found that a combination of comprehensive care and preventive treatment for adult migraine patients may be the best choice for migraine improvement. Adequate prevention is especially critical in reducing disability and preventing migraines from evolving. \\#4American migraine prevalence: Compared with episodic migraine, patients with chronic migraine have significantly lower family income levels, and patients with chronic migraine are about twice as likely to suffer from depression, anxiety, and chronic pain[45]. In addition to the difference in the frequency of headache attacks, chronic migraine, and paroxysmal migraine are also the key to distinguish between the two. $₫ \mathbf{5}$ preempt clinical program: The PREEMPT study for migraine prevention and treatment includes two phases, PREEMPT 1 and PREEMPT 2, which are used to evaluate the effectiveness, safety, and tolerability of botulinum toxin for preventing migraine in adults. The

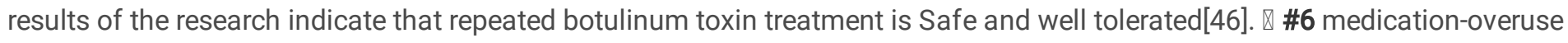
headache: This category mainly includes abnormal cortical responses to somatosensory stimulation in patients with drug overuse, risk factors, headache management, and personality characteristics of drug overuse headaches and episodic headaches. Coppola G's research[47] suggests that overuse of non-steroidal anti-inflammatory drugs In patients, the somatosensory cortex becomes more and more sensitive, which is conducive to compulsive drug intake behavioral sensitization; Sances G[48] believes that the negative prognostic factors for headache recurrence include intake of more than 30 doses per month, smoking, drinking, etc., which are dangerous Factors may influence treatment strategies. Tassorelli C[49] advocated that the consensus solutions proposed in different countries and different medical models have been proven effective for headaches caused by drug overuse. Sances G [50] found that MMPI-2 tracked the psychological characteristics of headache patients well, but he was not sure whether it was related to MMPI 2 "itself". $\otimes$ \#7 genome-wide association study: In his genome-wide association study, Anttila V [51] identified that the minor allele of rs1835740 on chromosome 8q22.1 was associated with migraine, and also found that rs 1835740 was the first genetic risk factor for migraine, which provided evidence for understanding the disease from the genome-wide perspective. Cluster categories that are not discussed in the article indicate that this category has no special significance, or that there are few related documents in this category, or that no similar research reports have been found.

\section{Cited references' cluster analysis comparison}

The most cited references in the two different periods of 2000-2009 and 2010-2019 are Silberstein SD (2004) [19] and Bes A (2013) [21] respectively. Among them, Silberstein SD's research is to understand the pathophysiological mechanism of migraine in the middle and late stages of migraine from the perspective of neuronal hyperexcitability, neurogenic inflammation, and peripheral and central sensitization, Bes A's research focuses on the international classification of headache diseases. Moreover, the most important literature of the two periods is Welch KMA (2001) [20] and Goadsby PJ (2017) [22]. Welch KMA's study found that the Periaqueductal gray matter may play a role as a "producer" in migraine attacks, while Goadsby PJ's study showed that 
the monthly subcutaneous injection of erenumab $70 \mathrm{mg}$ or $140 \mathrm{mg}$ could significantly reduce the frequency of migraine attacks and cut down the use of specific drugs for acute migraine within 6 months.

According to the cluster analysis of cited references, the common research in the two periods is chronic migraine. The difference between the two is: the study of chronic migraine in 2000-2009 mainly focused on the diagnostic criteria of the disease[52] and topiramate can decrease the headache days of patients with chronic migraine[53], while 2010-2019 focused on the disability, quality of life, sociodemographic[45] and comorbidity characteristics[54] of patients with chronic and intermittent migraine. By comparing \#6 Migraine headache in 2000-2009 with \#11 familial migraine in 2010-2019, the research content is mainly for familial hemiplegic migraine, but the previous period involved mainly gene mutations[55] and genes Localization[56], chromosome markers, and the latter mainly focused on genome-wide associations, confirming that the minor allele of rs 1835740 on chromosome 8q22.1 is related to migraine[57]. The similarities and differences between the two periods in terms of neuronal channels are: A Ducros's study[58] found that hemiplegic migraine caused by mutations in CACNA1A (a gene encoding neuronal calcium channels) has a broad clinical spectrum, including paroxysmal and permanent. Signs, the severity of the two are very different, and Martin Dichgans's research [59]found that a new site of familial hemiplegic migraine was found on chromosome 2q24. Sequencing of candidate genes in this region revealed a heterozygous missense mutation (GIn1489Lys) in the neuronal voltage-gated sodium channel gene SCN1A, which is related to epilepsy. The same mutation appeared in three familial hemiplegic migraine families. It results in a charge-change amino acid exchange in the so-called hinge domain of the protein, which is essential for the rapid inactivation of the channel.

Cluster analysis of cited references from 2000 to 2009: 欧0 comparative review: Mainly include the following aspects, the comparison of different drugs in the treatment of migraine (oral rizatriptan and sumatriptan in the acute treatment of migraine[60]), the same drug in the treatment of migraine in different aspects (Clinical efficacy and tolerability of $2.5 \mathrm{mg}$ zolmitriptan in the acute treatment of migraine[61]), and the comparison of different doses of different drugs in the treatment of migraine (crossover of $5 \mathrm{mg}$ and $10 \mathrm{mg}$ rizatriptan and $25 \mathrm{mg}$ and $50 \mathrm{mg}$ sumatriptan in the treatment of migraine Compare[62]). These studies provide us with methodological reference for randomized controlled trials of drug treatment on migraine from

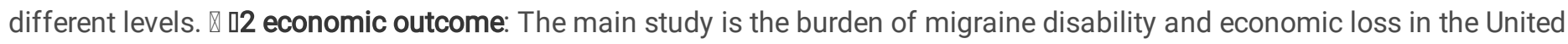
States[63]. The bed rest time of migraine patients takes up the working day and impairs work function, causing US employers to lose about 13 billion U.S. dollars and the annual direct cost of care is about 1 billion U.S. dollars. Therefore, the economic burden of migraine is mainly concentrated in bed rest and productivity declines, which in turn greatly affects employers' economic income, various screening and treatment options should be evaluated to reduce the burden of disease. $\nabla \square 5$ headache sufferers study: Mainly include the prevalence of migraine in different countries, the diagnosis mode, and preventive treatment of migraine. It was found that in the past ten years, the prevalence of migraine in France was $11.2 \%$ for women and $4.0 \%$ for men[64]. In the United States[65], the prevalence of migraine was $18.2 \%$ for women and $6.5 \%$ for men. The MIDAS score is lower than the score obtained in the US population study. This is helpful for us to understand the epidemiological results of migraine in different countries and to judge the clinical characteristics. $\mathbb{\square} \mathbf{8}$ chronic daily headache: This category of research mainly focuses on the epidemiological analysis of chronic daily headaches in the general population and the related factors of onset and relief. Castillo J's research[66] found that almost $5 \%$ of the general population suffers from chronic daily headaches. The proportions of chronic tension-type headaches and conversion migraines are very similar. A.I. Scher's study[67] confirms that chronic daily headaches are more common in women and have lower levels of education. It is more common among low-income people, white people, and married people. Its prevalence is related to obesity, arthritis, and diabetes. 如 9 trigeminovascular system: The main research in this category is that the intrinsic brain activity in the migraine model triggers trigeminal meningeal afferents. Hayrunnisa Bolay's research[68] found that the trigeminal nerve innervates the meninges and participates in migraine. Cortical diffusion inhibition leads to selective and persistent enhancement of blood flow in the middle meningeal artery, which provides a basis for the mechanism of migraine and a good interpretation of vasodilation in migraine. $\mathbb{\mathbf { Q } 1 0}$ using rizatriptan: The research in this category mainly shows solicitude for the application of rizatriptan in the acute treatment of migraine. Rizatriptan produces a consistently high response rate in the acute treatment of migraine attacks[69]. Comparing with sumatriptan, naratriptan, and zolmitriptan, it was found that rizatriptan eliminated a significant proportion of patients' disability within 2 hours. It provides a basis for the selection of drugs for migraine treatment. 如11 to-left shunt: The research in this category mainly concentrated on the relationship between migraine with aura and cardiac shunt from right to left, and the relationship between patent foramen ovale or atrial septal defect and the incidence of migraine. First of all, P Wilmshurst's research [70, 71]shows that there is a 
causal link between the right-to-left shunt of the heart and migraine with aura, and the closure of atrial defects can improve or eliminate migraine. Secondly, Babak Azarbal's study[72] further confirmed that occlusion of patent foramen ovale or atrial septal defect via catheter can completely relieve migraine symptoms in $60 \%$ of patients, providing a new method for the treatment of

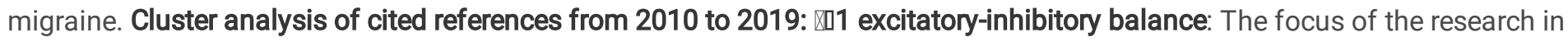
this category lies in the clinical relevance of cortical diffusion inhibition in neurological diseases[73] (including migraine). The cortex of migraine patients is thought to be over-excited due to impaired habit, which may explain why the patient Increased susceptibility to cortical diffusion inhibition, therefore, patients with familial hemiplegic migraine may show a particularly low threshold for cortical diffusion inhibition. $\otimes \square 3$ new analysis: The research in this category mainly includes the following aspects: updated guidelines for drug treatment to prevent adult-onset migraine[74], the latest statistical analysis of the prevalence and burden of migraine and severe headache in the United States[75], and evidence of acute treatment of adult migraine Evaluation[76]. The results show that valproate sodium, valproate sodium, topiramate, metoprolol, propranolol, and timolol are effective in preventing migraine, and can reduce the frequency and severity of migraine attacks; In the United States, the prevalence of migraine is very high, about one in seven people suffer from this disease each year. Migraine and headache are the main reasons for outpatient and emergency visits; triptans, ergotamine derivatives, NSAIDs, opioids, and combination drugs Effective for acute treatment of migraine. These studies have not only conducted in-depth discussions on migraine from the perspective of epidemiology but also the perspective of disease prevention and treatment, to guide clinicians in the precise treatment of migraine. $\otimes \square 5$ placebo effect: Studies in this category are mainly randomized, double-blind, placebo-controlled researches. The drugs involved mainly include botulinum toxin[43] and topiramate[53]. Botulinum toxin sodium has obvious advantages in curative effect, reducing disability, and improving function and related quality of life. Topiramate can significantly reduce the number of days of migraine per month, which provides medication guidance for the prevention of migraine. $\mathbb{\square} \mathbf{6}$ emerging drug: This category of research is mainly based on randomized controlled trials of human monoclonal antibodies in the prevention and treatment of paroxysmal migraine. The drugs involved mainly include fremanezumab[77] and erenumab[22]. Subcutaneous injection of fremanezumab can reduce the number of migraine days per month by an average of 1.3 to 1.5 days within 12 weeks. Subcutaneous injection of $70 \mathrm{mg}$ or $140 \mathrm{mg}$ of orrenzumab per month can significantly reduce the frequency of attacks and reduce the use of specific drugs, which provides a basis for the prevention and treatment of migraine from the perspective of monoclonal antibody. $\mathbf{\square 8} \mathbf{8}$ calcium channel function: Familial hemiplegic migraine type 1 calcium channel mutations cause increased cortical neurotransmission excitability, indicating that the destruction of excitatory-inhibitory balance and neuronal overactivity is the basis for migraine attacks susceptible to cortical diffusion inhibition ignition[78]. . $\mathrm{kg}$ key player neuropeptide: This category mainly introduces the pathophysiological basis of calcitonin gene-related peptide and its receptor triggering migraine[ $[79,80]$. This peptide is found in small and medium-sized neurons of the trigeminal ganglion and is released from the sensory nerves. Therefore, it is related to the pain pathway. It is released during migraine attacks and can cause migraines. It provides us with ideas for the development of new drugs to treat diseases from our understanding of this molecule.

\section{Keywords' cluster analysis comparison}

By comparing the cluster analysis of keywords in the two time periods, we found that the following research categories to-left shunt, chronic migraine, and familial hemiplegic migraine have all been analyzed in other cluster analysis, so we only discussed the cluster categories that did not appear. Cluster analysis of keywords from 2000 to 2009: $₫ 1$ trigeminal ganglion cell: In this category, the expression of $5-\mathrm{HT}_{1 \mathrm{~B}}$ receptor in rat trigeminal ganglion cell subtypes was mainly studied, $5-\mathrm{HT}_{1 \mathrm{~B}}$ receptor agonists can reduce neurogenic vasodilation by activating this receptor, which helps explain the anti-migraine drugs and accelerate the

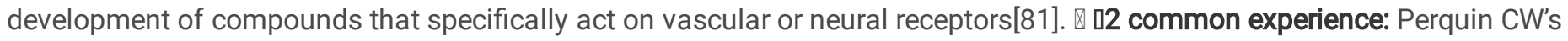
research[82] concentrates on the clinical epidemiology of pain in children and adolescents. Research has shown that chronic pain (including migraine) is a common problem in children and adolescents, especially severe chronic pain and multiple pain in girls 12 years and older. The high incidence of the disease should become the main focus of future health care services. The enlightenment for follow-up research is to focus on the pain process, quality of life, and biological-psychological-social factors over the years, which will be potential targets for intervention. 吅 3 cytokine level: This type of research is represented by the plasma cytokine levels of migraine patients. By collecting the plasma of migraine patients and healthy people, using enzymelinked immunosorbent assay to detect cytokine levels, the study found that tumor necrosis factor-alpha (TNFa), IL-1 $\beta$, and IL-10 may be involved in the onset of migraine[83]. $\mathbb{\square 4}$ sumatriptan tablet: This category mainly analyzes the cost-effectiveness of sumatriptan tablets and conventional therapies in the treatment of migraine. The results of the study show that the net benefit of

Page 21/34 
sumatriptan in treating a single migraine attack is $\$ 50$, while the net benefit of conventional therapy is $\$ 20$. And compared with the annual increase in net income, sumatriptan is 114-540 US dollars per patient more than conventional treatment. It further demonstrates that the advantages of sumatriptan in reducing the use of medical resources and the loss of productivity offset the price difference[84]. Cluster analysis of keywords from 2010 to 2019: $\otimes \square 2$ tension-type headache: Studies in this category included the association of multiple sclerosis with tension-type headache and migraine and the characteristics and chronicity of the two types of headache. Kister l's research[85] believes that migraine is earlier than multiple sclerosis, which may be one of the risk factors for multiple sclerosis, and it may also be its early clinical manifestations. Ashina $\mathrm{S}^{\prime}$ study[86] argues that the chronicity of primary headache involves various risk factors. Combining these risk factors with some of the clinical features of the two types of headaches can predict the cause of the increased frequency and chronicity of primary headaches. $\otimes \square 3$ transient global amnesia: This category analyzes the relationship between migraine and transient global amnesia through a cohort study. The results of the study show that migraine patients are at increased risk of transient global amnesia, especially women aged 4060 [87], which provided a valuable method for the association between migraine and other diseases. $\mathrm{x} 5 \mathrm{5}$ migraine education: This category mainly recommends the impact of migraine primary care education program on headache and the benefits of quality of life. The research results exhibit that Mercy Migraine Management Program has a significant effect in improving headache frequency, cognitive and emotional aspects of headache treatment, which offers in-depth research power for migraine prevention and health care[88]. $\otimes \square 7$ laser-evoked potential: The research that was absorbed in this category is laser evoked potential. In de Tommaso M's research[89], high-frequency repetitive transcranial magnetic stimulation was utilized to detect the effect of the left primary motor cortex on subjective pain and evoked response induced by laser stimulation in contralateral hand and supraorbital region of patients with migraine without aura in a critical period. The results demonstrate that high frequency repetitive transcranial magnetic stimulation of the motor cortex has a better effect on injury than other pain regulation methods, and its potential advantages should not be ignored. $₫ \square 8$ anxiety symptom: The focus of research in this category is that migraine recurrence is not related to depression or anxiety. Through a multicenter randomized double-blind trial study, Mitsikostas DD [90] found that the recurrence of migraine was not affected by the Hamilton Depression Scale or Anxiety Score, SCL-90-R score or treatment, indicating that depression or anxiety symptoms did not affect the recurrence of migraine in the drug treatment of acute migraine.

\section{Comparison of research hotspots}

Comparison of keyword research hotspots in the two periods: The research hotspot during 2000-2009 was "stimulation", which mainly included transcranial magnetic stimulation[91], vagus nerve stimulation[92], visual stimulation[93], light stimulation[94], laser pain stimulation[95], etc. The main effects of these stimulations are focused on the treatment of migraine, treatments such as vagus nerve stimulation for refractory migraine, occipital nerve stimulation for chronic migraine, and transcranial magnetic stimulation (including single pulse and double pulse forms) for migraine, all of which enrich the treatment of migraine. Moreover, the research hotspot in 2010-2019 is "triptan"(Most of them start from the treatment of migraine[96]), including the first and second-generation triptans. Specific drugs include sumatriptan, zolmitriptan, rizatriptan, naratriptan, eletriptan, fluvatriptan, etc. The incidence of side effects And the severity is lower than traditional anti-migraine drugs. These studies provide evidence for the clinically reasonable and safe use of paroxysmal migraine.

\section{Conclusions}

In this paper, we used Citespace to conduct bibliometric analysis on migraine in the Web of Science database from 2000 to 2019 and acquired the knowledge mapping of countries, institutions, authors, keywords, and Cited references in 2000-2009 and 2010-2019. This study provides a new perspective on the trend of migraine. Although this study has some limitations, it fully reveals the global trend of migraine. The overall trend of publications is increasing year by year, and the growth rate in 20002009 is greater than that in 2010-2019. Developed countries still occupy the dominant position, but some developing countries, such as China, Southeast Asia, and the Middle East, are developing rapidly. Lipton RB is the most important author in migraine research, not only because he is the most prolific author, but also because he has a high centrality value. From 2000 to 2009 , the most important reference was the study "Periaqueductal gray matter dysfunction in migraine: cause or the burden of illness?" published by Welch KMA in Headache in 2001, and the most frequently cited is Silberstein SDA's "migration pathology and its clinical" published in cephalalgia in 2004; In 2010-2019, the most key reference was the "A Controlled Trial of Erenumab for 
Episodic Migraine" study published by Godsby PJ in 2017 in the New England Journal of Medicine. The most cited document is the "The International Classification of Headache Disorders, 3rd edition (beta version)" study published by Bes A in Cephalalgia in 2013. And the most convincing research topic is prevalence from 2000 to 2019 . Comparing the $S$ values of different cluster analysis to get the most reliable research category. In 2000-2009, the most credible research included potential anti-migraine agents, reducing headache recurrence, to-left shunt, spectrum study, while in 2010-2019, the most convincing researches are chronic migration prevention, following symptomatic treatment, placebo controlled phase and family study hemiplegic migraine. According to burst analysis, the hotspots of keywords are "stimulation" in 2000-2009, while the hotspots in 2010-2019 are mainly on "triptan" therapy for chronic daily headache.

All in all, the results of this study may provide researchers with useful information, such as research frontiers, potential collaborators and cooperative institutions, important research content, and key references.

\section{Research Limitations}

There are two limitations to this study. Firstly, although the search strategy searches for synonyms of the MeSH subject words in PubMed, it may still cause some pieces of literature to be missed. Secondly, Citespace's function of removing duplication has certain limitations, which may bias the research results. It is just an analysis of the Web of Science database, which may not fully represent the overall trend. Also, because some literature will be published online in advance, it will cause the inconsistency between the collection date and the publication date of this literature, so the criteria we include need to be further improved.

\section{Abbreviations}

SCIE=Science Citation Index Expanded; WOS=Web of Science; Albert Einstein Coll Med= Albert Einstein College of Medicine; Harvard Univ= Harvard University; Univ Roma La Sapienza =University of Roma-La Sapienza; Univ Copenhagen =University of Copenhagen; Leiden Univ= Leiden University; New England Ctr Headache= New England Center for Headache; Mayo Clin= Mayo clinicUniv Calif Los Angeles=University of California, Los Angeles; Inst Neurol =Institute of Neurology; Thomas Jefferson Univ=Thomas Jefferson University; UCL Natl Hosp Neurol \& Neurosurg=UCL National Hospital for Neurology and Neurosurgery; Univ Essen Gesamthsch= University of Essen; Univ Cincinnati= University of Cincinnati; Univ Liege= University of Liège; Merck \& Co Inc= Merck \& Co., Inc; Kings Coll London = King's College London; Univ Calif San Francisco= University of California San Francisco; Harvard Med Sch= Harvard Medical School; Univ Pavia=University of Pavia; Montefiore Med Ctr=Montefiore Medical Center; Amgen Inc=Amgen Inc; Univ Sao Paulo=Universidade de Saõ Paulo; Houston Headache Clin=Houston Headache Clinic; Duke Univ=Duke University; Diamond Headache Clin Ltd=Diamond Headache Clinic; Lipton=Lipton; Ortho McNeil Janssen Sci Affairs LLC= Ortho-McNeil Janssen Scientific Affairs LLC; Univ Erlangen Nurnberg=University of Erlangen; Michigan Head Pain \& Neurol Inst=Michigan Head Pain and Neurological Institute; Drexel Univ=Drexel University; Vedanta Res=Vedanta Research; Montefiore Headache Ctr=Montefiore Headache Center; Novartis Pharma AG=Novartis Pharma AG; Sapienza Univ Rome=Sapienza University of Rome; Sapienza Univ=Sapienza University; Charite=Charite; Univ Mississippi=University of Mississippi; Cincinnati Childrens Hosp Med Ctr=Cincinnati Children's Hospital Medical Center

\section{Declarations}

\section{Acknowledgments}

The authors would like to express their appreciation to Professor Chaomei Chen for inventing the CiteSpace and making it free to use.

\section{Funding}

This study was supported by grants from the Shanghai Science and Technology Commission, "science and technology innovation action plan" clinical medicine project (no. 18401971200), Shanghai 13th five years plan key clinical specialty construction project (no. shslczdzk04901). Scientific research project of Putuo Hospital, Shanghai University of Traditional Chinese Medicine (no. 2020304A). Budget Project of Shanghai University of Traditional Chinese Medicine (no. 2019WK118).

Page 23/34 


\section{Author Contributions}

YZ designed this study. LH performed the search. YZ collected data. LH and WL rechecked data. YZ and LH performed analysis. HG and ML: critically revised the work. YZ and LH have contributed equally to this work and should be considered co-first authors.

\section{Disclosure}

The authors report no conflicts of interest in this work.

\section{References}

1. Gazerani P (2020) Migraine and Diet. NUTRIENTS 12 (6)

2. Pelzer N, Louter MA, van Zwet EW, Nyholt DR, Ferrari MD, van den Maagdenberg AM, Haan J, Terwindt GM (2019) Linking migraine frequency with family history of migraine. CEPHALALGIA 39 (2):229-236

3. Burch RC, Buse DC, Lipton RB (2019) Migraine: Epidemiology, Burden, and Comorbidity. NEUROL CLIN 37 (4):631-649

4. Lagman-Bartolome AM, Lay C (2019) Migraine in Women. NEUROL CLIN 37 (4):835-845

5. Youssef PE, Mack KJ (2020) Episodic and chronic migraine in children. DEV MED CHILD NEUROL 62 (1):34-41

6. Wijeratne T, Tang HM, Crewther D, Crewther S (2019) Prevalence of Migraine in the Elderly: A Narrated Review. NEUROEPIDEMIOLOGY 52 (1-2):104-110

7. Parikh SK, Young WB (2019) Migraine: Stigma in Society. Curr Pain Headache Rep 23 (1):8

8. Silberstein SD, Lee L, Gandhi K, Fitzgerald T, Bell J, Cohen JM (2018) Health care Resource Utilization and Migraine Disability Along the Migraine Continuum Among Patients Treated for Migraine. HEADACHE 58 (10):1579-1592

9. Zhou S, Tao Z, Zhu Y, Tao L (2019) Mapping theme trends and recognizing hot spots in postmenopausal osteoporosis research: a bibliometric analysis. PEERJ 7:e8145

10. Tao L, Zhou S, Tao Z, Wen K, Da W, Meng Y, Zhu Y (2020) The publication trends and hot spots of scoliosis research from 2009 to 2018: a 10-year bibliometric analysis. Ann Transl Med 8 (6):365

11. Li S, Wang H, Zheng H, Li N, Sun C, Meng X, Zheng W, Wang K, Qin H, Gao W, Shen Z (2020) Bibliometric Analysis of Pediatric Liver Transplantation Research in PubMed from 2014 to 2018. Med Sci Monit 26:e922517

12. Zhu X, Niu X, Li T, Liu C, Chen L, Tan G (2019) Identification of research trends concerning application of stent implantation in the treatment of pancreatic diseases by quantitative and biclustering analysis: a bibliometric analysis. PEERJ 7:e7674

13. Zheng K, Wang X (2019) Publications on the Association Between Cognitive Function and Pain from 2000 to 2018: A Bibliometric Analysis Using CiteSpace. Med Sci Monit 25:8940-8951

14. Qi B, Jin S, Qian H, Zou Y (2020) Bibliometric Analysis of Chronic Traumatic Encephalopathy Research from 1999 to 2019. Int J Environ Res Public Health 17 (15)

15. Mao Z, Liu C, Chen S, Zhu ZG, Kang HJ, Zhou FH (2016) A bibliometric analysis of exertional heat stroke research in Web of Science. Mil Med Res 3:31

16. Ke L, Lu C, Shen R, Lu T, Ma B, Hua Y (2020) Knowledge Mapping of Drug-Induced Liver Injury: A Scientometric Investigation (2010-2019). FRONT PHARMACOL 11:842

17. Wang SQ, Gao YQ, Zhang C, Xie YJ, Wang JX, Xu FY (2020) A Bibliometric Analysis Using CiteSpace of Publications from 1999 to 2018 on Patient Rehabilitation After Total Knee Arthroplasty. Med Sci Monit 26:e920795

18. Miao Y, Liu R, Pu Y, Yin L (2017) Trends in esophageal and esophagogastric junction cancer research from 2007 to 2016 : A bibliometric analysis. Medicine (Baltimore) 96 (20):e6924

19. Silberstein SD (2004) Migraine pathophysiology and its clinical implications. CEPHALALGIA 24 Suppl 2:2-7

20. Welch KM, Nagesh V, Aurora SK, Gelman N (2001) Periaqueductal gray matter dysfunction in migraine: cause or the burden of illness? HEADACHE 41 (7):629-637 
21. Bes A, Kunkel R, Lance J, Nappi G, Pfaffenrath V, Rose F, Ducros A (2013) The International Classification of Headache Disorders, 3rd edition (beta version). CEPHALALGIA 33 (9):629-808

22. Goadsby PJ, Reuter U, Hallstrom Y, Broessner G, Bonner JH, Zhang F, Sapra S, Picard H, Mikol DD, Lenz RA (2017) A Controlled Trial of Erenumab for Episodic Migraine. N Engl J Med 377 (22):2123-2132

23. Zhao Y, Huang L, Xiang M, Li Q, Miao W, Lou Z (2018) Trends in conjunctivochalasis research from 1986 to 2017: A bibliometric analysis. Medicine (Baltimore) 97 (39):e12643

24. Gladstone JP, Eross EJ, Dodick DW (2004) Migraine in special populations. Treatment strategies for children and adolescents, pregnant women, and the elderly. POSTGRAD MED 115 (4):39-44, 47-50

25. Lipton RB, Scher Al, Kolodner K, Liberman J, Steiner TJ, Stewart WF (2002) Migraine in the United States: epidemiology and patterns of health care use. NEUROLOGY 58 (6):885-894

26. Wang SJ, Fuh JL, Young YH, Lu SR, Shia BC (2000) Prevalence of migraine in Taipei, Taiwan: a population-based survey. CEPHALALGIA 20 (6):566-572

27. O'Brien HL, Kabbouche MA, Hershey AD (2010) Treatment of acute migraine in the pediatric population. Curr Treat Options Neurol 12 (3):178-185

28. Stuginski-Barbosa J, Dach F, Bigal M, Speciali JG (2012) Chronic pain and depression in the quality of life of women with migraine-a controlled study. HEADACHE 52 (3):400-408

29. Stewart WF, Lipton RB, Chee E, Sawyer J, Silberstein SD (2000) Menstrual cycle and headache in a population sample of migraineurs. NEUROLOGY 55 (10):1517-1523

30. Silberstein SD, Massiou H, Le Jeunne C, Johnson-Pratt L, McCarroll KA, Lines CR (2000) Rizatriptan in the treatment of menstrual migraine. OBSTET GYNECOL 96 (2):237-242

31. Luciani R, Carter D, Mannix L, Hemphill M, Diamond M, Cady R (2000) Prevention of migraine during prodrome with naratriptan. CEPHALALGIA 20 (2):122-126

32. Afra J, Proietti CA, Sandor PS, Schoenen J (2000) Comparison of visual and auditory evoked cortical potentials in migraine patients between attacks. CLIN NEUROPHYSIOL 111 (6):1124-1129

33. Swan SK, Alcorn HJ, Rodgers A, Hustad CM, Ramsey KE, Woll S, Skobieranda F (2006) Pharmacokinetic profile of rizatriptan 10-mg tablet and 10-mg orally disintegrating tablet administered with or without water in healthy subjects: an open-label, randomized, single-dose, 3-period crossover study. J CLIN PHARMACOL 46 (2):172-178

34. Grazzi L, Andrasik F, D'Amico D, Leone M, Usai S, Kass SJ, Bussone G (2002) Behavioral and pharmacologic treatment of transformed migraine with analgesic overuse: outcome at 3 years. HEADACHE 42 (6):483-490

35. Grazzi L, Andrasik F, D'Amico D, Usai S, Kass S, Bussone G (2004) Disability in chronic migraine patients with medication overuse: treatment effects at 1-year follow-up. HEADACHE 44 (7):678-683

36. Schurks M, Zee RY, Buring JE, Kurth T (2008) Interrelationships among the MTHFR 677C>T polymorphism, migraine, and cardiovascular disease. NEUROLOGY 71 (7):505-513

37. Kurth T, Gaziano JM, Cook NR, Logroscino G, Diener HC, Buring JE (2006) Migraine and risk of cardiovascular disease in women. JAMA 296 (3):283-291

38. Scher Al, Stewart WF, Buse D, Krantz DS, Lipton RB (2008) Major life changes before and after the onset of chronic daily headache: a population-based study. CEPHALALGIA 28 (8):868-876

39. Bigal ME, Sheftell FD, Rapoport AM, Tepper SJ, Lipton RB (2002) Chronic daily headache: identification of factors associated with induction and transformation. HEADACHE 42 (7):575-581

40. van den Broek RW, MaassenVanDenBrink A, de Vries R, Bogers AJ, Stegmann AP, Avezaat CJ, Saxena PR (2000) Pharmacological analysis of contractile effects of eletriptan and sumatriptan on human isolated blood vessels. EUR $J$ PHARMACOL 407 (1-2):165-173

41. Kors EE, Vanmolkot KR, Haan J, Kheradmand KS, Stroink H, Laan LA, Gill DS, Pascual J, van den Maagdenberg AM, Frants RR, Ferrari MD (2004) Alternating hemiplegia of childhood: no mutations in the second familial hemiplegic migraine gene ATP1A2. NEUROPEDIATRICS 35 (5):293-296

Page 25/34 
42. Haan J, Kors EE, Terwindt GM, Vermeulen FL, Vergouwe MN, van den Maagdenberg AM, Gill DS, Pascual J, Ophoff RA, Frants RR, Ferrari (2000) Alternating hemiplegia of childhood: no mutations in the familial hemiplegic migraine CACNA1A gene. CEPHALALGIA 20 (8):696-700

43. Aurora SK, Dodick DW, Turkel CC, DeGryse RE, Silberstein SD, Lipton RB, Diener HC, Brin MF (2010) OnabotulinumtoxinA for treatment of chronic migraine: results from the double-blind, randomized, placebo-controlled phase of the PREEMPT 1 trial. CEPHALALGIA 30 (7):793-803

44. Pompili M, Serafini G, Innamorati M, Serra G, Dominici G, Fortes-Lindau J, Pastina M, Telesforo L, Lester D, Girardi P, Tatarelli R, Martelletti P (2010) Patient outcome in migraine prophylaxis: the role of psychopharmacological agents. Patient Relat Outcome Meas 1:107-118

45. Buse DC, Manack A, Serrano D, Turkel C, Lipton RB (2010) Sociodemographic and comorbidity profiles of chronic migraine and episodic migraine sufferers. J Neurol Neurosurg Psychiatry 81 (4):428-432

46. Diener HC, Dodick DW, Aurora SK, Turkel CC, DeGryse RE, Lipton RB, Silberstein SD, Brin MF (2010) OnabotulinumtoxinA for treatment of chronic migraine: results from the double-blind, randomized, placebo-controlled phase of the PREEMPT 2 trial. CEPHALALGIA 30 (7):804-814

47. Coppola G, Curra A, Di Lorenzo C, Parisi V, Gorini M, Sava SL, Schoenen J, Pierelli F (2010) Abnormal cortical responses to somatosensory stimulation in medication-overuse headache. BMC NEUROL 10:126

48. Sances G, Ghiotto N, Galli F, Guaschino E, Rezzani C, Guidetti V, Nappi G (2010) Risk factors in medication-overuse headache: a 1-year follow-up study (care II protocol). CEPHALALGIA 30 (3):329-336

49. Tassorelli C, Jensen R, Allena M, De Icco R, Sances G, Katsarava Z, Lainez M, Leston J, Fadic R, Spadafora S, Pagani M, Nappi G (2014) A consensus protocol for the management of medication-overuse headache: Evaluation in a multicentric, multinational study. CEPHALALGIA 34 (9):645-655

50. Sances G, Galli F, Anastasi S, Ghiotto N, De Giorgio G, Guidetti V, Firenze C, Pazzi S, Quartesan R, Gallucci M, Nappi G (2010) Medication-overuse headache and personality: a controlled study by means of the MMPI-2. HEADACHE 50 (2):198-209

51. Anttila V, Stefansson H, Kallela M, Todt U, Terwindt GM, Calafato MS, Nyholt DR, Dimas AS, Freilinger T, Muller-Myhsok B, Artto V, Inouye M, Alakurtti K, Kaunisto MA, Hamalainen E, de Vries B, Stam AH, Weller CM, Heinze A, Heinze-Kuhn K, Goebel I, Borck G, Gobel H, Steinberg S, Wolf C, Bjornsson A, Gudmundsson G, Kirchmann M, Hauge A, Werge T, Schoenen J, Eriksson JG, Hagen K, Stovner L, Wichmann HE, Meitinger T, Alexander M, Moebus S, Schreiber S, Aulchenko YS, Breteler MM, Uitterlinden AG, Hofman A, van Duijn CM, Tikka-Kleemola P, Vepsalainen S, Lucae S, Tozzi F, Muglia P, Barrett J, Kaprio J, Farkkila M, Peltonen L, Stefansson K, Zwart JA, Ferrari MD, Olesen J, Daly M, Wessman M, van den Maagdenberg AM, Dichgans M, Kubisch C, Dermitzakis ET, Frants RR, Palotie A (2010) Genome-wide association study of migraine implicates a common susceptibility variant on 8q22.1. NAT GENET 42 (10):869-873

52. Olesen J, Bousser MG, Diener HC, Dodick D, First M, Goadsby PJ, Gobel H, Lainez MJ, Lance JW, Lipton RB, Nappi G, Sakai F, Schoenen J, Silberstein SD, Steiner TJ (2006) New appendix criteria open for a broader concept of chronic migraine. CEPHALALGIA 26 (6):742-746

53. Diener HC, Bussone G, Van Oene JC, Lahaye M, Schwalen S, Goadsby PJ (2007) Topiramate reduces headache days in chronic migraine: a randomized, double-blind, placebo-controlled study. CEPHALALGIA 27 (7):814-823

54. Buse DC, Silberstein SD, Manack AN, Papapetropoulos S, Lipton RB (2013) Psychiatric comorbidities of episodic and chronic migraine. J NEUROL 260 (8):1960-1969

55. Kraus RL, Sinnegger MJ, Glossmann H, Hering S, Striessnig J (1998) Familial hemiplegic migraine mutations change alpha1A Ca2+ channel kinetics. J BIOL CHEM 273 (10):5586-5590

56. Joutel A, Bousser MG, Biousse V, Labauge P, Chabriat H, Nibbio A, Maciazek J, Meyer B, Bach MA, Weissenbach J, Et A (1993) A gene for familial hemiplegic migraine maps to chromosome 19. NAT GENET 5 (1):40-45

57. Anttila V, Stefansson H, Kallela M, Todt U, Terwindt GM, Calafato MS, Nyholt DR, Dimas AS, Freilinger T, Muller-Myhsok B, Artto V, Inouye M, Alakurtti K, Kaunisto MA, Hamalainen E, de Vries B, Stam AH, Weller CM, Heinze A, Heinze-Kuhn K, Goebel I, Borck G, Gobel H, Steinberg S, Wolf C, Bjornsson A, Gudmundsson G, Kirchmann M, Hauge A, Werge T, Schoenen J, Eriksson JG, Hagen K, Stovner L, Wichmann HE, Meitinger T, Alexander M, Moebus S, Schreiber S, Aulchenko YS, Breteler MM, 
Uitterlinden AG, Hofman A, van Duijn CM, Tikka-Kleemola P, Vepsalainen S, Lucae S, Tozzi F, Muglia P, Barrett J, Kaprio J, Farkkila M, Peltonen L, Stefansson K, Zwart JA, Ferrari MD, Olesen J, Daly M, Wessman M, van den Maagdenberg AM, Dichgans M, Kubisch C, Dermitzakis ET, Frants RR, Palotie A (2010) Genome-wide association study of migraine implicates a common susceptibility variant on 8q22.1. NAT GENET 42 (10):869-873

58. Ducros A, Denier C, Joutel A, Cecillon M, Lescoat C, Vahedi K, Darcel F, Vicaut E, Bousser MG, Tournier-Lasserve E (2001) The clinical spectrum of familial hemiplegic migraine associated with mutations in a neuronal calcium channel. $N$ Engl $\mathrm{J}$ Med $345(1): 17-24$

59. Dichgans M, Freilinger T, Eckstein G, Babini E, Lorenz-Depiereux B, Biskup S, Ferrari MD, Herzog J, van den Maagdenberg AM, Pusch M, Strom TM (2005) Mutation in the neuronal voltage-gated sodium channel SCN1A in familial hemiplegic migraine. LANCET 366 (9483):371-377

60. Tfelt-Hansen P, Teall J, Rodriguez F, Giacovazzo M, Paz J, Malbecq W, Block GA, Reines SA, Visser WH (1998) Oral rizatriptan versus oral sumatriptan: a direct comparative study in the acute treatment of migraine. Rizatriptan 030 Study Group. HEADACHE 38 (10):748-755

61. Solomon GD, Cady RK, Klapper JA, Earl NL, Saper JR, Ramadan NM (1997) Clinical efficacy and tolerability of $2.5 \mathrm{mg}$ zolmitriptan for the acute treatment of migraine. The 042 Clinical Trial Study Group. NEUROLOGY 49 (5):1219-1225

62. Goldstein J, Ryan R, Jiang K, Getson A, Norman B, Block GA, Lines C (1998) Crossover comparison of rizatriptan 5 mg and $10 \mathrm{mg}$ versus sumatriptan $25 \mathrm{mg}$ and $50 \mathrm{mg}$ in migraine. Rizatriptan Protocol 046 Study Group. HEADACHE 38 (10):737-747

63. Hu XH, Markson LE, Lipton RB, Stewart WF, Berger ML (1999) Burden of migraine in the United States: disability and economic costs. Arch Intern Med 159 (8):813-818

64. Henry P, Auray JP, Gaudin AF, Dartigues JF, Duru G, Lanteri-Minet M, Lucas C, Pradalier A, Chazot G, El HA (2002) Prevalence and clinical characteristics of migraine in France. NEUROLOGY 59 (2):232-237

65. Lipton RB, Stewart WF, Diamond S, Diamond ML, Reed M (2001) Prevalence and burden of migraine in the United States: data from the American Migraine Study II. HEADACHE 41 (7):646-657

66. Castillo J, Munoz P, Guitera V, Pascual J (1999) Kaplan Award 1998. Epidemiology of chronic daily headache in the general population. HEADACHE 39 (3):190-196

67. Scher Al, Stewart WF, Ricci JA, Lipton RB (2003) Factors associated with the onset and remission of chronic daily headache in a population-based study. PAIN 106 (1-2):81-89

68. Bolay H, Reuter U, Dunn AK, Huang Z, Boas DA, Moskowitz MA (2002) Intrinsic brain activity triggers trigeminal meningeal afferents in a migraine model. NAT MED 8 (2):136-142

69. Goadsby PJ (2000) Rizatriptan in acute treatment of migraine: update on new comparative data. CEPHALALGIA 20 SuppI 1:10-15

70. Wilmshurst PT, Nightingale S, Walsh KP, Morrison WL (2000) Effect on migraine of closure of cardiac right-to-left shunts to prevent recurrence of decompression illness or stroke or for haemodynamic reasons. LANCET 356 (9242):1648-1651

71. Wilmshurst P, Nightingale S (2001) Relationship between migraine and cardiac and pulmonary right-to-left shunts. Clin Sci (Lond) 100 (2):215-220

72. Azarbal B, Tobis J, Suh W, Chan V, Dao C, Gaster R (2005) Association of interatrial shunts and migraine headaches: impact of transcatheter closure. J AM COLL CARDIOL 45 (4):489-492

73. Lauritzen M, Dreier JP, Fabricius M, Hartings JA, Graf R, Strong AJ (2011) Clinical relevance of cortical spreading depression in neurological disorders: migraine, malignant stroke, subarachnoid and intracranial hemorrhage, and traumatic brain injury. J Cereb Blood Flow Metab 31 (1):17-35

74. Silberstein SD, Holland S, Freitag F, Dodick DW, Argoff C, Ashman E (2012) Evidence-based guideline update: pharmacologic treatment for episodic migraine prevention in adults: report of the Quality Standards Subcommittee of the American Academy of Neurology and the American Headache Society. NEUROLOGY 78 (17):1337-1345

75. Burch RC, Loder S, Loder E, Smitherman TA (2015) The prevalence and burden of migraine and severe headache in the United States: updated statistics from government health surveillance studies. HEADACHE 55 (1):21-34 
76. Marmura MJ, Silberstein SD, Schwedt TJ (2015) The acute treatment of migraine in adults: the american headache society evidence assessment of migraine pharmacotherapies. HEADACHE 55 (1):3-20

77. Dodick DW, Silberstein SD, Bigal ME, Yeung PP, Goadsby PJ, Blankenbiller T, Grozinski-Wolff M, Yang R, Ma Y, Aycardi E (2018) Effect of Fremanezumab Compared With Placebo for Prevention of Episodic Migraine: A Randomized Clinical Trial. JAMA 319 (19):1999-2008

78. Tottene A, Conti R, Fabbro A, Vecchia D, Shapovalova M, Santello M, van den Maagdenberg AM, Ferrari MD, Pietrobon D (2009) Enhanced excitatory transmission at cortical synapses as the basis for facilitated spreading depression in $\mathrm{Ca}(\mathrm{v}) 2.1$ knockin migraine mice. NEURON 61 (5):762-773

79. Russell FA, King R, Smillie SJ, Kodji X, Brain SD (2014) Calcitonin gene-related peptide: physiology and pathophysiology. PHYSIOL REV 94 (4):1099-1142

80. Ho TW, Edvinsson L, Goadsby PJ (2010) CGRP and its receptors provide new insights into migraine pathophysiology. NAT REV NEUROL 6 (10):573-582

81. Wotherspoon G, Priestley JV (2000) Expression of the 5-HT1B receptor by subtypes of rat trigeminal ganglion cells. NEUROSCIENCE 95 (2):465-471

82. Perquin CW, Hazebroek-Kampschreur AA, Hunfeld JA, Bohnen AM, van Suijlekom-Smit LW, Passchier J, van der Wouden JC (2000) Pain in children and adolescents: a common experience. PAIN 87 (1):51-58

83. Perini F, D'Andrea G, Galloni E, Pignatelli F, Billo G, Alba S, Bussone G, Toso V (2005) Plasma cytokine levels in migraineurs and controls. HEADACHE 45 (7):926-931

84. Biddle AK, Shih YC, Kwong WJ (2000) Cost-benefit analysis of sumatriptan tablets versus usual therapy for treatment of migraine. PHARMACOTHERAPY 20 (11):1356-1364

85. Kister I, Caminero AB, Herbert J, Lipton RB (2010) Tension-type headache and migraine in multiple sclerosis. Curr Pain Headache Rep 14 (6):441-448

86. Ashina S, Lyngberg A, Jensen R (2010) Headache characteristics and chronification of migraine and tension-type headache: A population-based study. CEPHALALGIA 30 (8):943-952

87. Lin KH, Chen YT, Fuh JL, Li SY, Chen TJ, Tang CH, Wang SJ (2014) Migraine is associated with a higher risk of transient global amnesia: a nationwide cohort study. EUR J NEUROL 21 (5):718-724

88. Smith TR, Nicholson RA, Banks JW (2010) Migraine education improves quality of life in a primary care setting. HEADACHE 50 (4):600-612

89. de Tommaso M, Brighina F, Fierro B, Francesco VD, Santostasi R, Sciruicchio V, Vecchio E, Serpino C, Lamberti P, Livrea P (2010) Effects of high-frequency repetitive transcranial magnetic stimulation of primary motor cortex on laser-evoked potentials in migraine. J HEADACHE PAIN 11 (6):505-512

90. Mitsikostas DD, Vikelis M, Kodounis A, Zaglis D, Xifaras M, Doitsini S, Georgiadis G, Thomas A, Charmoussi S (2010) Migraine recurrence is not associated with depressive or anxiety symptoms. Results of a randomized controlled trial. CEPHALALGIA 30 (6):690-695

91. Fumal A, Bohotin V, Vandenheede M, Schoenen J (2003) Transcranial magnetic stimulation in migraine: a review of facts and controversies. ACTA NEUROL BELG 103 (3):144-154

92. Mauskop A (2005) Vagus nerve stimulation relieves chronic refractory migraine and cluster headaches. CEPHALALGIA 25 (2):82-86

93. Backer M, Sander D, Hammes MG, Funke D, Deppe M, Conrad B, Tolle TR (2001) Altered cerebrovascular response pattern in interictal migraine during visual stimulation. CEPHALALGIA 21 (5):611-616

94. Ances BM, Detre JA (2003) Perfusion changes with photic stimulation during two phases of the menstrual cycle: a pilot study comparing controls and true menstrual migraine patients. CEPHALALGIA 23 (9):907-913

95. de Tommaso M, Marinazzo D, Stramaglia S (2005) The measure of randomness by leave-one-out prediction error in the analysis of EEG after laser painful stimulation in healthy subjects and migraine patients. CLIN NEUROPHYSIOL 116

(12):2775-2782

96. Loder E (2010) Triptan therapy in migraine. N Engl J Med 363 (1):63-70

Page 28/34 
Figures
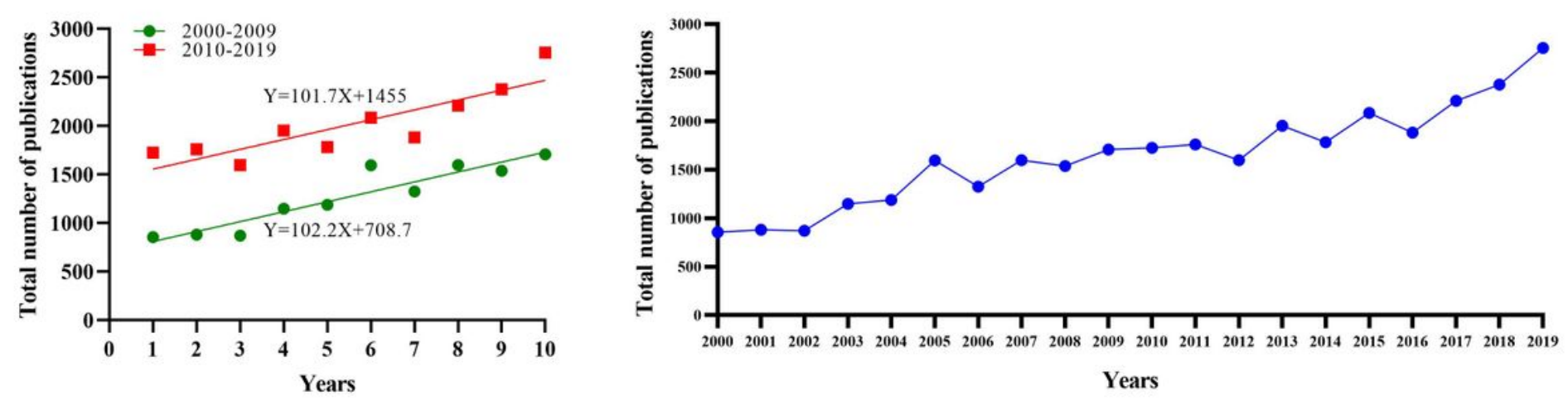

\section{Figure 1}

Line Chart of the total number of publications on migraine The abscissa in the figure represents the year and the ordinate represents the total number of publications.
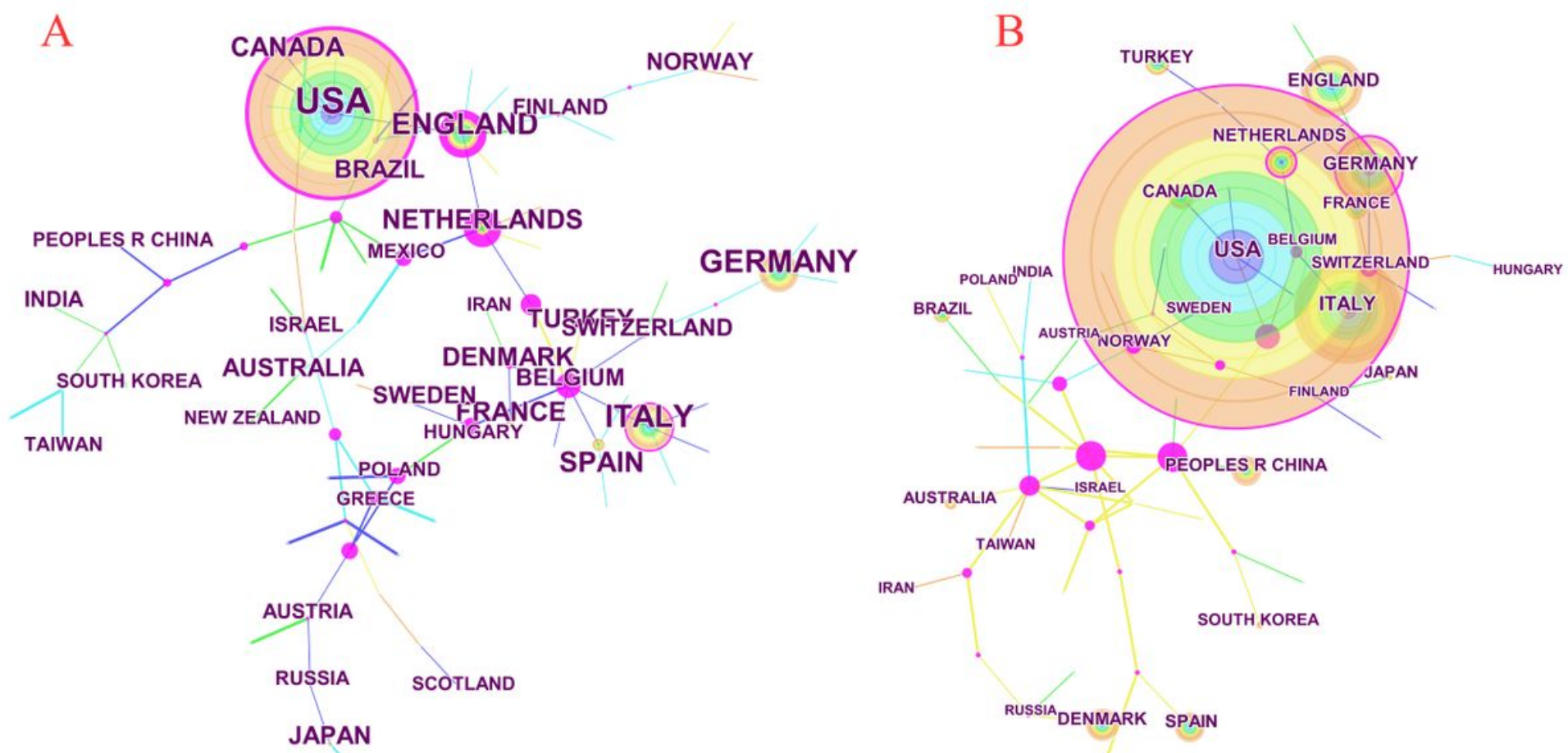

\section{Figure 2}

Network of countries/territories on migraine The purple node in the middle of the annual ring means the influence and the significance of a country/territory. The larger the node and the more purple it exhibits, the greater is the importance of the country/territory. 

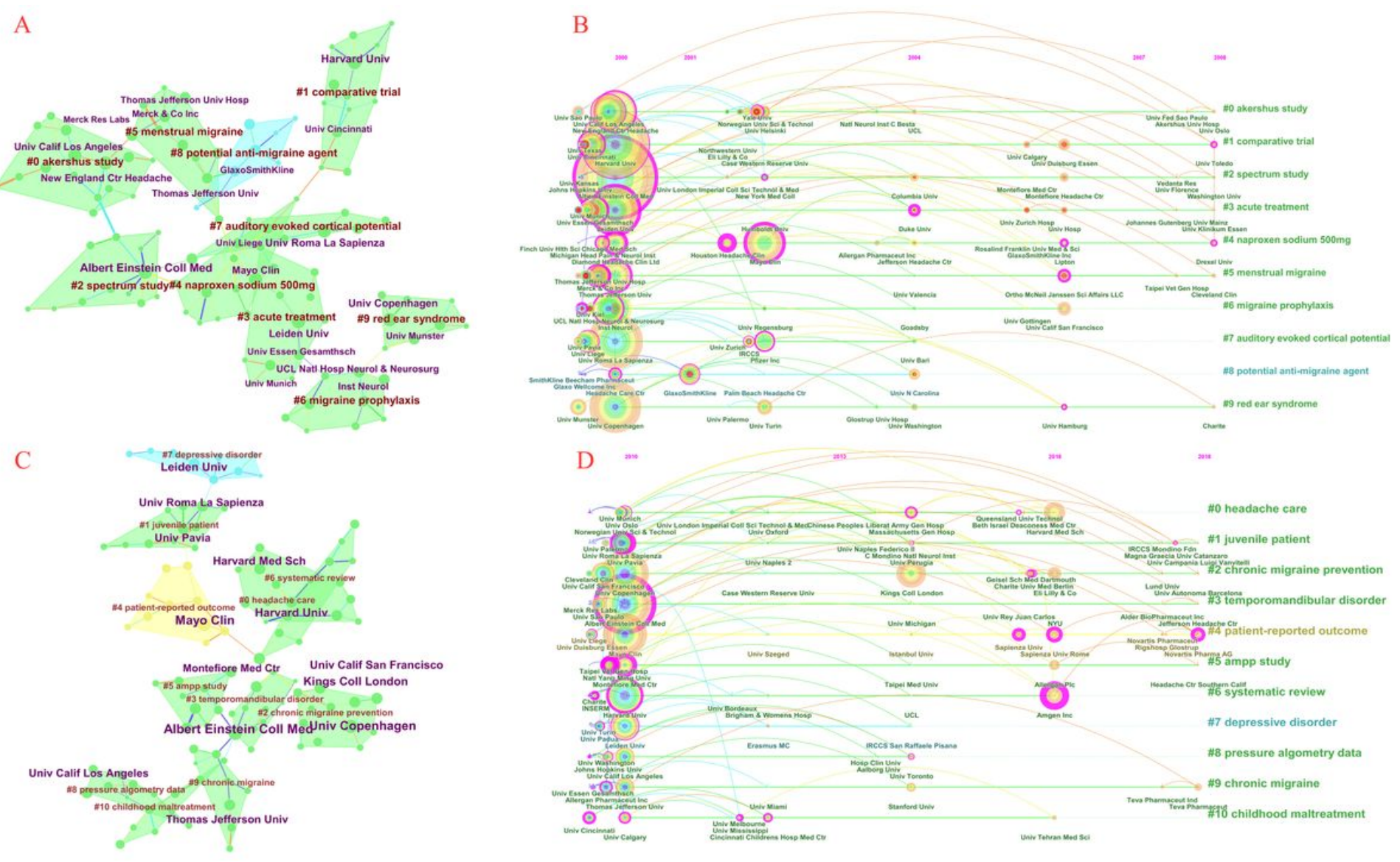

\section{Figure 3}

$3(A)$ and 3(C) Cluster map of institutions based on label clusters with title terms. The combination of symbols and numbers delegates the institutions' study of similar categories. $3(B)$ and $3(D)$ Timeline zone of institutions on migraine. The purple node in the middle of the annual ring means the influence and the significance of the institution. Institutions with the homologous research category are on the same time horizon. 


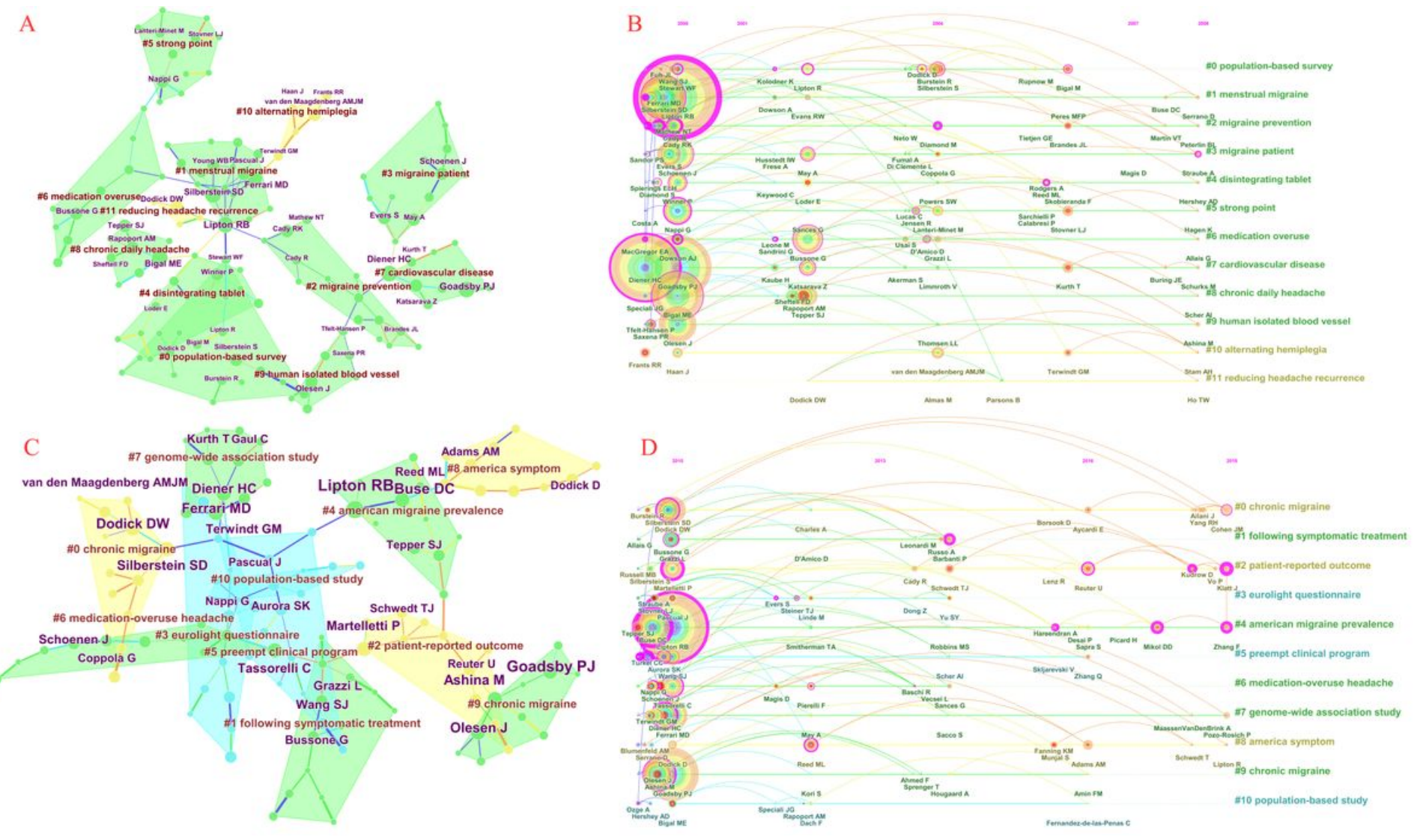

Figure 4

4(A) and 4(C) Cluster map of authors based on label clusters with title terms. The combination of symbols and numbers delegates the authors' study of similar categories. 4(B) and 4(D) Timeline zone of authors on migraine. 


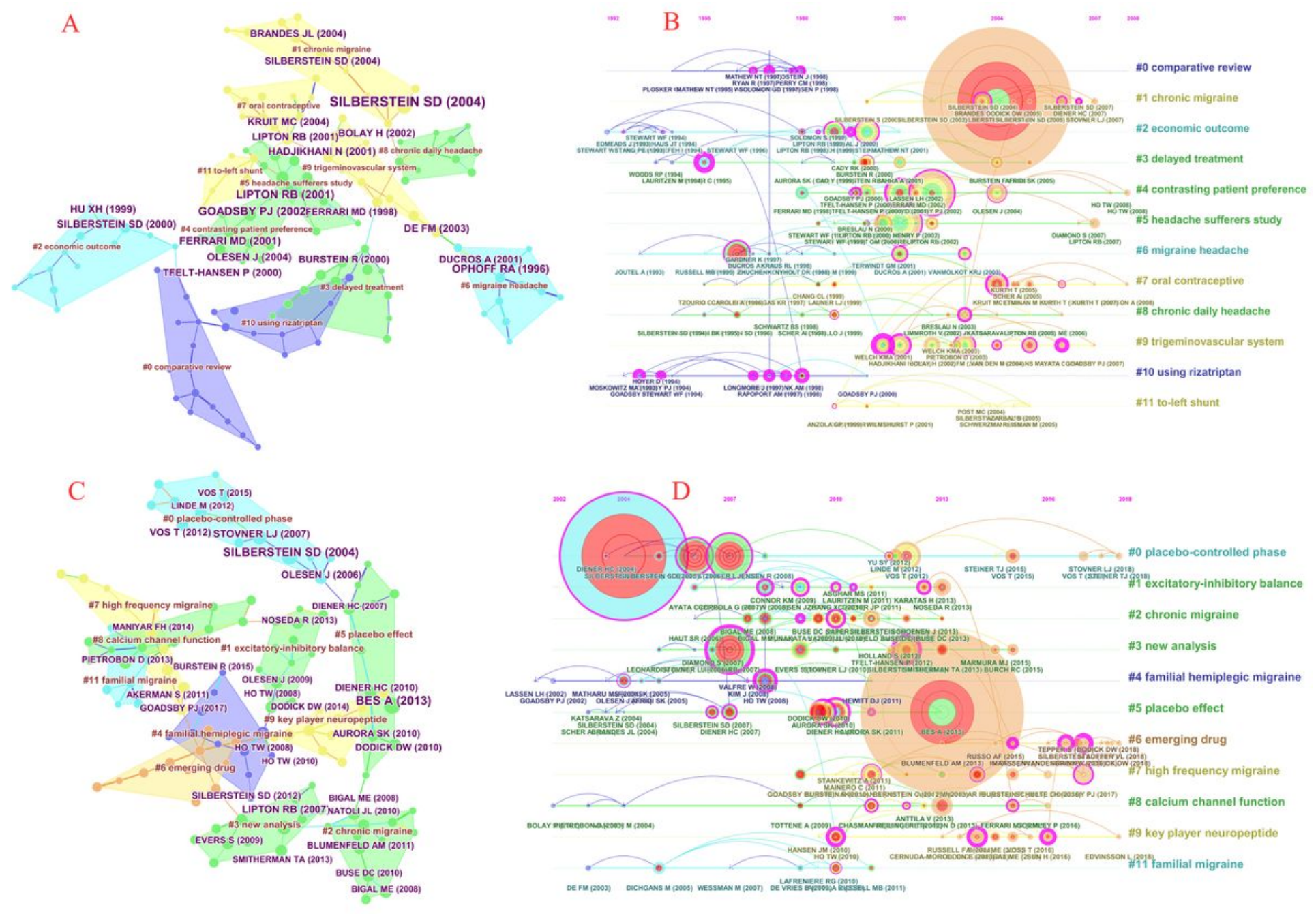

\section{Figure 5}

5(A) and 5(C) Cluster map of Cited Reference based on label clusters with title terms. The combination of symbols and numbers delegates the Cited References' study of similar categories. 5(B) and 5(D) Timeline zone of Cited Reference on migraine. 
A

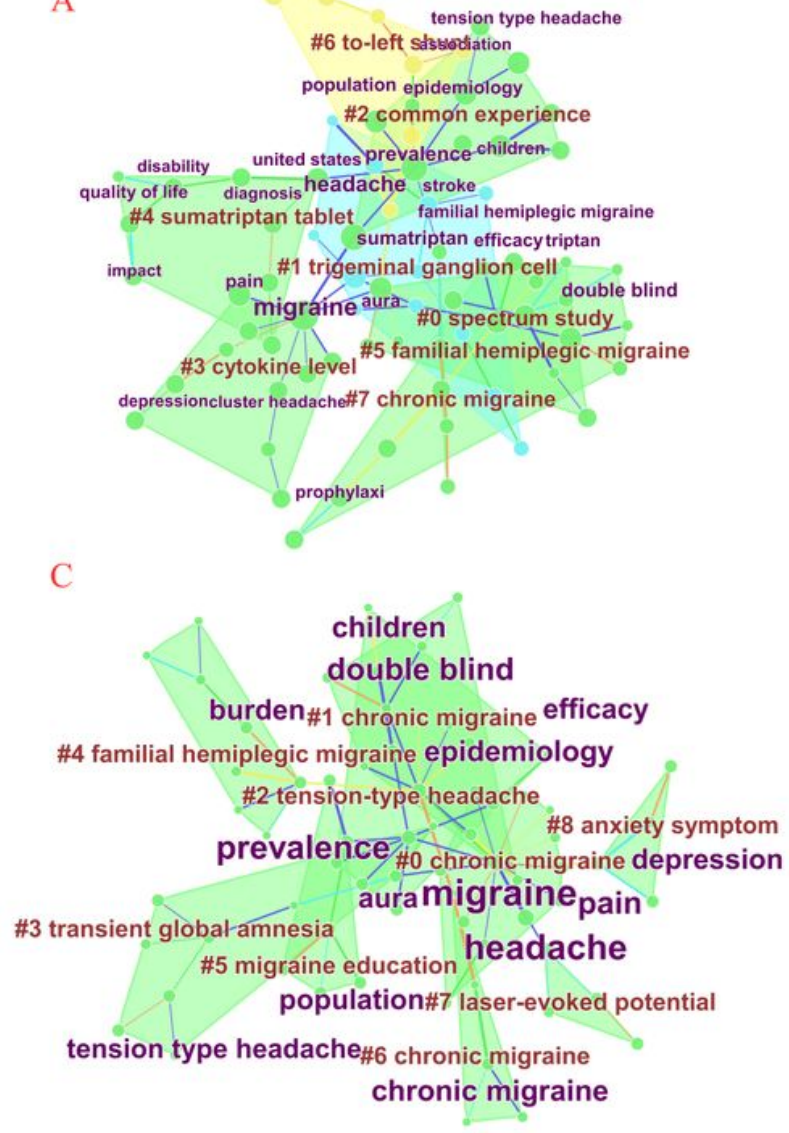

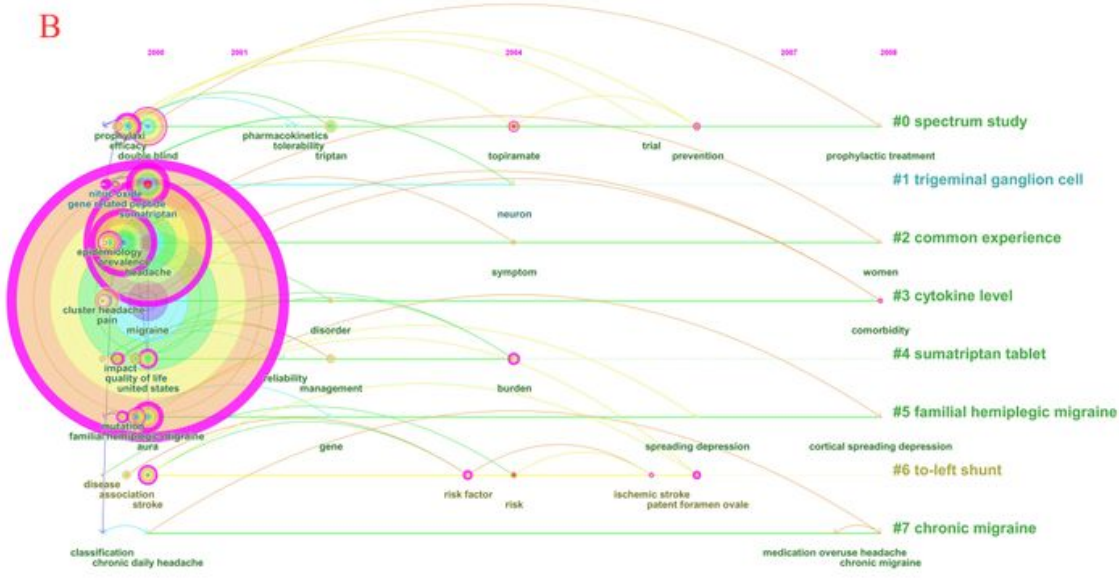

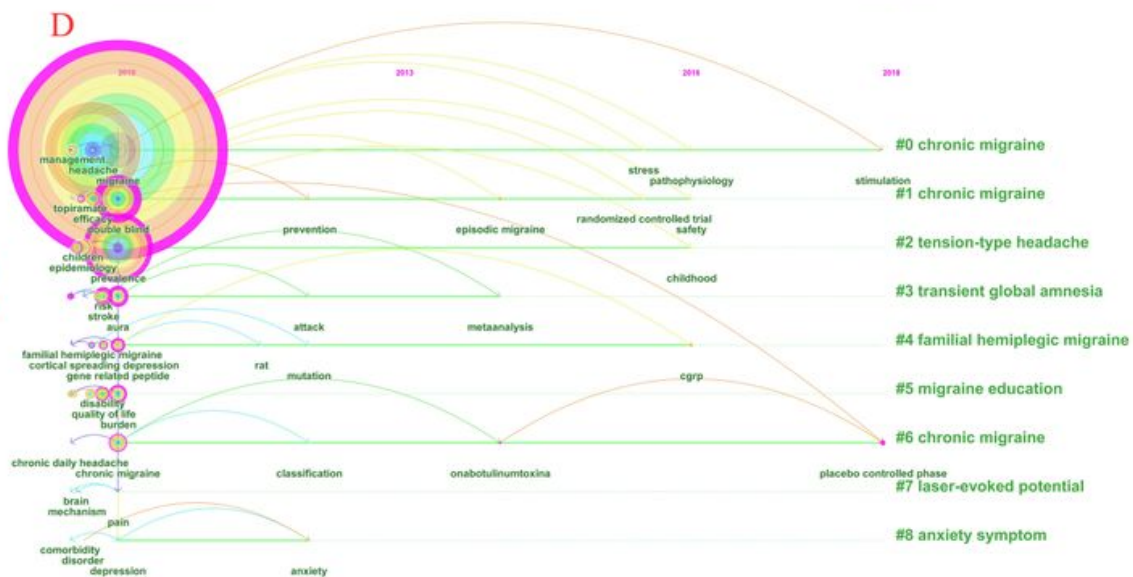

\section{Figure 6}

6(A) and 6(C) Cluster map of Keyword based on label clusters with title terms. The combination of symbols and numbers delegates the Keywords' study of similar categories. 6(B) and 6(D) Timeline zone of Keyword on migraine. 


\section{A Top 30 Keywords with the Strongest Citation Bursts}

\begin{tabular}{|c|c|c|c|c|}
\hline Keywords & Year & Strength Begin & $2000-2009$ & Keywords \\
\hline rizatriptan & 2000 & 36.21212000 & $2003=$ & familial hemiplegic migraine \\
\hline serotonin & 2000 & $35.8122 \mathbf{2 0 0 0}$ & 2003 - & nitric oxide \\
\hline safety & 2000 & 25.43032000 & 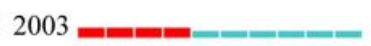 & therapy \\
\hline attack & 2000 & 13.51232000 & $2001=$ & chronic daily headache \\
\hline stimulation & 2000 & 20.55172000 & $2005=$ & triptan \\
\hline subcutaneous sumatriptan & 2000 & 23.79582000 & $2001=$ & trial \\
\hline calcium channel & 2000 & 15.48202000 & $2001=$ & spreading depression \\
\hline zolmitriptan & 2000 & 21.02302000 & $2001=$ & patent foramen ovale \\
\hline receptor & 2000 & 16.03582000 & 2001 & placebo \\
\hline cerebral blood flow & 2000 & 28.62162000 & $2003=$ & sumatriptan \\
\hline activation & 2000 & 14.03672000 & $2003=$ & rat \\
\hline substance $p$ & 2000 & 16.58972000 & $2001=$ & classification \\
\hline sumatriptan & 2000 & 39.27652000 & 2002 & mutation \\
\hline classification & 2000 & 28.25302001 & $2005=$ & attack \\
\hline reliability & 2000 & 19.91142002 & 2003 & onabotulinumtoxina \\
\hline pharmacokinetics & 2000 & $18.8330 \quad 2002$ & $2003-\ldots=$ & metaanalysis \\
\hline gene & 2000 & $18.8330 \mathbf{2 0 0 2}$ & $2003-1=$ & stress \\
\hline tolerability & 2000 & 14.95632002 & 2006 - & chronic pain \\
\hline care & 2000 & 17.75492002 & 2003 & childhood \\
\hline neuron & 2000 & 23.86002004 & - & cgrp \\
\hline triptan & 2000 & $7.8425 \quad 2004$ & 2005 & randomized controlled trial \\
\hline rat & 2000 & 19.20152004 & - $-1-1$ & pathophysiology \\
\hline tension-type headache & 2000 & 2.94722005 & 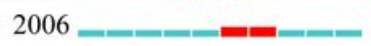 & episodic migraine \\
\hline trial & 2000 & 25.95942006 & 2007 - & safety \\
\hline patent foramen ovale & 2000 & 27.98022006 & $2009=$ & \\
\hline prevention & 2000 & 29.37982006 & 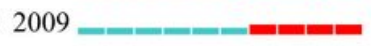 & \\
\hline ischemic stroke & 2000 & 23.98702006 & 2009 - & \\
\hline topiramate & 2000 & 13.13072006 & ש-10 & \\
\hline spreading depression & 2000 & 20.28212006 & $2007-1-1-2=$ & \\
\hline risk & 2000 & 11.35062006 & 2009 ב- 2 & \\
\hline
\end{tabular}

\section{B Top 24 Keywords with the Strongest Citation Bursts}

Year Strength Begin End 2010 - 2019

201020.009620102013

$2010 \quad 35.991920102011$

$2010 \quad 31.880420102015$

201032.451020102015

$2010 \quad 37.59172010 \quad 2015$

201033.90082010201

201031.81032010201

$2010 \quad 47.218320102013$

201032.332920102011

$2010 \quad 38.422220102014$

$2010 \quad 30.79012012 \quad 2013$

$2010 \quad 34.83752012 \quad 2013$

$2010 \quad 31.80172012 \quad 2013$

$201038.40062012 \quad 2015$

$2010 \quad 16.675920142015$

$2010 \quad 9.034920152016$

201032.865220162017

$2010 \quad 35.021820162019$

$2010 \quad 33.356820162017$

$2010 \quad 54.394220162019$

201035.517320162019

$2010 \quad 58.629220162019$

$201038.71402017 \quad 2019$

$2010 \quad 44.66342017 \quad 2019$

\section{Figure 7}

7(A) and 7(B) Top 30 and Top 24 references with the strongest citation bursts. The red bars represent some references cited frequently; the green bars represent references cited infrequently. 